\title{
ANALYSIS OF NOISE POLLUTION LEVEL IN MAJOR AREAS OF HYDERABAD CITY, INDIA
}

\author{
K. Sri laxmi \\ Department of Chemistry \\ St Anns College for Women-Mehdipatnam, \\ Hyderabad, Telangana
}

\author{
Khairunnisa Amreen \\ Department of Chemistry \\ St Anns College for Women-Mehdipatnam \\ Hyderabad, Telangana
}

\begin{abstract}
Noise pollution, despite of being under rated, it has become a major concern in the growing nations. The urbanization is the major cause leading to high decibels. Owing to this everyday increasing problem, the government is taking all possible initiatives to cut down the noise levels especially due to vehicles. However, the urban and metro cities are still badly suffering. In this report, an attempt is made to record noise levels in heavy traffic areas of Hyderabad city. The analysis was done during the month of November 2018. Thirty major heavy traffic zones were chosen for the study. Using a simple noise meter, measurement of noise for three different days: a working day, a week day and Diwali day was carried out. Two different time intervals were chosen for recording; morning 9:45 to 10:00 am, evening 6:30 to $6: 45 \mathrm{pm}$ and on Diwali day evening 7:30 to 7:45 pm. The meter was set to record the noise level for every second during the exposure time of $\mathbf{1 5}$ minutes. It was found that the noise level in most of the zones increased more than the standard prescribed values and on the eve of Diwali there was an extensive noise recorded.
\end{abstract}

Keywords - Noise pollution, Hyderabad city, traffic areas, noise meter

\section{INTRODUCTION}

In developing countries like India, industrialization is at the peak to meet the rapid increasing demands of growing population every day [1]. Urbanization of the rural areas is at its pinnacle today. This is not only affecting the environment through water/air contamination and exhaustion of nonrenewable resources but also in terms of noise pollution [2]. Despite of many precautionary measures being adapted, cities in India like Delhi, Mumbai, Chennai, Kolkatta, Hyderabad, Bangalore, Pune etc. (metro cities and industrial hubs), are recorded to possess heavy noise pollution. Noise pollution is a measure of any unwanted sounds which causes a feeling of nausea and irritation[3,4]. Though it is a matter of serious concern and immediate action but most of the time it is overlooked due to the fact that it does not cause immediate damage or visible symptoms[5]. However, It is a very well known fact that long time exposure to high decibel, causes grievous heath related disorders like irritation, nervous break downs, cardio vascular, hypertensions, stress, sleeping disorder, hormonal imbalance etc.[6-8]. It is not just the humans who get affected with this but animals and plants as well. Many birds, mammals, amphibians, reptiles etc. are found to get effected due to noise produced by human activities [9]. A study has also shown that exposure of animals to heavy noise even effect there reproduction system [10]. Research also proves that, traffic noise can restrict the plant growth causing decrease in the plant height as compared to the plants with no exposure to traffic sounds $[11,12]$. If the problem of noise pollution remained unsolved, in near future, it would increase a lot more. Today, sounds from vehicles and traffic have become the primary cause of noise pollution. Besides these, activities like rallies, processions, celebrations, music bands, crackers, etc. during festivities are also very common source. India being home to diverse communities, various festivals are observed and celebrated lavishly without the environment concern. The government is trying to take possible initiatives as and when required to solve this issue. The basic measure taken in many areas in this regard is educating people and spreading awareness. Many non-governmental organizations are also involved. Surveys are also being conducted in many cities to analyze the causes and amount of noise in there so that appropriate measures can be taken. As a social responsibility initiative, we conducted the survey of noise pollution in Hyderabad city. Thirty different areas including heavy traffic areas, railway stations, bus stands, residential areas, silence zones like hospitals, schools etc. were chosen for the study. At the peak hours for a period of 15 minutes in the morning and evening, noise was recorded using a noise meter[1]. Survey was conducted on three different days, on Monday (working day), Sunday (Weekened), on the eve of Diwali celebration (Holiday), Diwali is a major festival of India which is celebrated each year in the month of November (Autumn) in high spirits. Bursting of crackers on the eve of Diwali as a part of celebration is a common practice being carried out from many years.

In order to compare the noise on different days, same timing and methodology was adapted. Following are the areas chosen to conduct the study: Charminar, Lad Bazaar, Afzal gunj, Mir alam mandi, Begum Bazaar, Sultan Bazaar, Kothi, Abids, 
Mehdipatnam, Nampally, Lakdi-ka-pool, Dilsukhnagar, Shaikpet, Gachibowli, Banjara Hills, Ameerpet, Bowenpally, Tirumalagiri, Marredpally, Lalaguda, Amberpet, Malakpet, Himayat Nagar, Langer house, Musheerabad, Secunderabad, Bahadurpura, Bandlaguda, Moosarambagh, Khairtabad. These places are mostly commercial and transportation areas and are reported to have heavy traffic.

\section{METHODOLOGY}

In these studies, some major areas of Hyderabad which are prone to heavy traffic and commercial spaces were chosen for the assessment. A simple noise meter was used for the studies. The noise level at the morning peak hours (9:45 to 10:00 am) and evening peak hours $(6: 30 \mathrm{pm}$ to $6: 45 \mathrm{pm})$ on working day and a weekend were recorded. In addition, the noise levels on the day of Diwali, morning (9:45 to 10:00 am) and evening (7:30 to $7: 45 \mathrm{pm}$ ) were also recorded for 15 minutes continuously. The data obtained is tabulated and average is taken. In further, graphs are plotted for each area to analyze the noise pollution in the chosen area.

\section{EXPERIMENT AND RESULT}

Noise level analysis was done for three different days: working, weekend and Diwali holiday at fixed timings: morning and evening peak hours were recorded. Thirty major areas of Hyderabad were chosen for the survey and noise level was noted by exposing noise meter for 15 minutes. Average noise level is recorded. All the areas showed a drastic increase in the $\mathrm{db}$ level on the eve of Diwali. Table 1 shows the average noise recorded data in various parts of Hyderabad.

Table 1: Comparison of Average Noise level (db) on Diwali evening Table 2-31 shows the detailed data of the noise recorded in various areas. It observed that during the peak morning hours of week day, the recorded noise is high $\mathrm{db}$ as compared to the same timing on weekends. Probably this is due to the fact the most of the offices, schools etc. are closed . On the other hand, the average noise recorded in weekend's evening is slightly high plausibly due to outings. Similarly, morning of Diwali is found to be a little high noise level in specially commercial on account of festivity. However, the eve of Diwali recorded heavy noise in all the surveyed areas.

\begin{tabular}{|c|c|c|}
\hline S.No. & Area & $\begin{array}{c}\text { Avg. noise (db) on Diwali } \\
\text { evening }\end{array}$ \\
\hline 1 & Charminar & 93.9 \\
\hline 2. & Lad Bazaar & 96.1 \\
\hline 3. & Afzal Gunj & 95.75 \\
\hline 4. & Mir Alam mandi & 99.6 \\
\hline 5. & Begum Bazaar & 99.3 \\
\hline 6. & Sultan Bazar & 97.7 \\
\hline 7. & Kothi & 98.95 \\
\hline 8 & Abids & 96.5 \\
\hline 9. & Mehdipatnam & 97.25 \\
\hline 10. & Nampally & 99.1 \\
\hline 11. & Lakdi-ka-pool & 97.7 \\
\hline 12. & Dilsukhnagar & 98.1 \\
\hline 13. & Shaikhpet & 99.75 \\
\hline 14. & Gachibowli & 93.3 \\
\hline 15. & Banjara Hills & 85.25 \\
\hline 16. & Ameerpet & 95.40 \\
\hline 17. & Bowenpally & 96.4 \\
\hline 18. & Tirumalagiri & 96.98 \\
\hline 19. & Marredpally & 97.11 \\
\hline 20. & Lalaguda & 98.4 \\
\hline 21. & Amberpet & 99.39 \\
\hline 22. & Malakpet & 97.59 \\
\hline 23. & Himayat Nagar & 96.39 \\
\hline 24. & Langer House & 95.80 \\
\hline 25. & Musheerabad & 96.39 \\
\hline 26. & Secunderabad & 99.39 \\
\hline 27. & Bahadurpura & 96.29 \\
\hline 28. & Bandlaguda & 94.39 \\
\hline 29. & Moosarambagh & 97.4 \\
\hline 30. & Khairtabad & 98.4 \\
\hline
\end{tabular}


Published Online July 2019 in IJEAST (http://www.ijeast.com)

Table 2: Noise level data near Charminar

\begin{tabular}{|c|c|c|c|c|c|c|c|c|c|c|c|c|}
\hline $\begin{array}{l}\text { S. } \\
\text { No. }\end{array}$ & $\begin{array}{l}\text { Morning } \\
\text { Peak } \\
\text { hours }\end{array}$ & $\begin{array}{l}\text { Noise } \\
\text { level } \\
\text { (db) }\end{array}$ & $\begin{array}{l}\text { Evening } \\
\text { peak } \\
\text { hours }\end{array}$ & $\begin{array}{l}\text { Noise } \\
\text { level } \\
\text { (db) }\end{array}$ & $\begin{array}{l}\text { Morning } \\
\text { Peak } \\
\text { hours }\end{array}$ & $\begin{array}{l}\text { Noise } \\
\text { level } \\
\text { (dB) }\end{array}$ & $\begin{array}{l}\text { Evening } \\
\text { peak } \\
\text { hours }\end{array}$ & $\begin{array}{l}\text { Noise } \\
\text { level } \\
\text { (dB) }\end{array}$ & $\begin{array}{l}\text { Morning } \\
\text { hours }\end{array}$ & $\begin{array}{l}\text { Noise } \\
\text { level } \\
\text { (dB) }\end{array}$ & $\begin{array}{l}\text { Evening } \\
\text { peak } \\
\text { hours }\end{array}$ & $\begin{array}{l}\text { Noise } \\
\text { level } \\
\text { (dB) }\end{array}$ \\
\hline & $\begin{array}{l}\text { (Working } \\
\text { Day) }\end{array}$ & & $\begin{array}{l}\text { (Working } \\
\text { Day) }\end{array}$ & & (Weekend) & & (Weekend) & & $\begin{array}{l}\text { (Diwali } \\
\text { holiday) }\end{array}$ & & $\begin{array}{l}\text { (Eve of } \\
\text { Diwali) }\end{array}$ & \\
\hline & $\begin{array}{l}\text { 9:45 am } \\
\text { to } 10: 00 \\
\text { am } \\
\end{array}$ & & $\begin{array}{l}6: 30 \mathrm{pm} \\
\text { to } 6: 45 \\
\text { pm } \\
\end{array}$ & & $\begin{array}{l}9: 45 \text { am to } \\
10: 00 \mathrm{am}\end{array}$ & & $\begin{array}{l}\text { 6:30 pm to } \\
6: 45 \mathrm{pm}\end{array}$ & & $\begin{array}{l}\text { 9:45 am } \\
\text { to } 10: 00 \\
\text { am } \\
\end{array}$ & & $\begin{array}{l}7: 30 \mathrm{pm} \\
\text { to } 7: 45 \\
\text { pm } \\
\end{array}$ & \\
\hline 1 & $9: 45$ & 69.2 & $6: 30$ & 76.2 & $9: 45$ & 62.1 & $6: 30$ & 79.5 & $9: 45$ & 75.2 & $7: 30$ & 93.5 \\
\hline 2 & $9: 46$ & 69.8 & $6: 31$ & 76.1 & $9: 46$ & 62.4 & $6: 31$ & 79.2 & $9: 46$ & 75.3 & $7: 31$ & 92.2 \\
\hline 3 & $9: 47$ & 69.2 & $6: 32$ & 77.6 & $9: 47$ & 62.2 & $6: 32$ & 79.4 & $9: 47$ & 76.1 & $7: 32$ & 93.6 \\
\hline 4 & $9: 48$ & 69.1 & $6: 33$ & 75.3 & $9: 48$ & 62.6 & $6: 33$ & 79.3 & $9: 48$ & 76.3 & $7: 33$ & 93.8 \\
\hline 5 & $9: 49$ & 70.5 & $6: 34$ & 75.6 & $9: 49$ & 62.4 & $6: 34$ & 79.6 & $9: 49$ & 76.9 & $7: 34$ & 93.1 \\
\hline 6 & $9: 50$ & 70.2 & $6: 35$ & 75.9 & $9: 50$ & 62.9 & $6: 35$ & 79.4 & $9: 50$ & 77.2 & $7: 35$ & 96.2 \\
\hline 7 & $9: 51$ & 70.2 & $6: 36$ & 75.2 & $9: 51$ & 63.6 & $6: 36$ & 80.2 & $9: 51$ & 77.6 & $7: 36$ & 93.2 \\
\hline 8 & $9: 52$ & 69.4 & $6: 37$ & 75.2 & $9: 52$ & 63.8 & $6: 37$ & 80.4 & $9: 52$ & 77.2 & $7: 37$ & 93.4 \\
\hline 9 & $9: 53$ & 70.5 & $6: 38$ & 75.9 & $9: 53$ & 63.1 & $6: 38$ & 81.2 & $9: 53$ & 77.6 & $7: 38$ & 95.5 \\
\hline 10 & $9: 54$ & 70.3 & $6: 39$ & 80.1 & $9: 54$ & 63.6 & $6: 39$ & 81.4 & $9: 54$ & 78.6 & $7: 39$ & 95.1 \\
\hline 11 & $9: 55$ & 71.6 & $6: 40$ & 81.9 & $9: 55$ & 64.4 & $6: 40$ & 81.9 & $9: 55$ & 78.5 & $7: 40$ & 95.2 \\
\hline 12 & $9: 56$ & 70.5 & $6: 41$ & 81.2 & $9: 56$ & 64.5 & $6: 41$ & 82.6 & $9: 56$ & 78.9 & $7: 41$ & 96.2 \\
\hline 13 & $9: 57$ & 71.6 & $6: 42$ & 80.3 & $9: 57$ & 64.9 & $6: 42$ & 83.2 & $9: 57$ & 79.6 & $7: 42$ & 95.1 \\
\hline 14 & $9: 58$ & 71.3 & $6: 43$ & 80.9 & $9: 58$ & 64.3 & $6: 43$ & 83.2 & $9: 58$ & 79.1 & $7: 43$ & 95.4 \\
\hline 15 & $9: 59$ & 71.2 & $6: 44$ & 81.1 & $9: 59$ & 63.2 & $6: 44$ & 82.4 & $9: 59$ & 79.2 & $7: 44$ & 95.9 \\
\hline \multirow[t]{2}{*}{16} & $10: 00$ & 71.9 & $6: 45$ & 83.3 & $10: 00$ & 65.2 & $6: 45$ & 82.9 & $10: 00$ & 80.3 & $7: 45$ & 94.3 \\
\hline & Average & 70.55 & & 79.75 & & 63.65 & & 81.2 & & $\mathbf{7 7 . 7 5}$ & & 93.9 \\
\hline
\end{tabular}


International Journal of Engineering Applied Sciences and Technology, 2019

Vol. 4, Issue 3, ISSN No. 2455-2143, Pages 573-605

Published Online July 2019 in IJEAST (http://www.ijeast.com)

Table 3: Noise level data at lad Bazaar

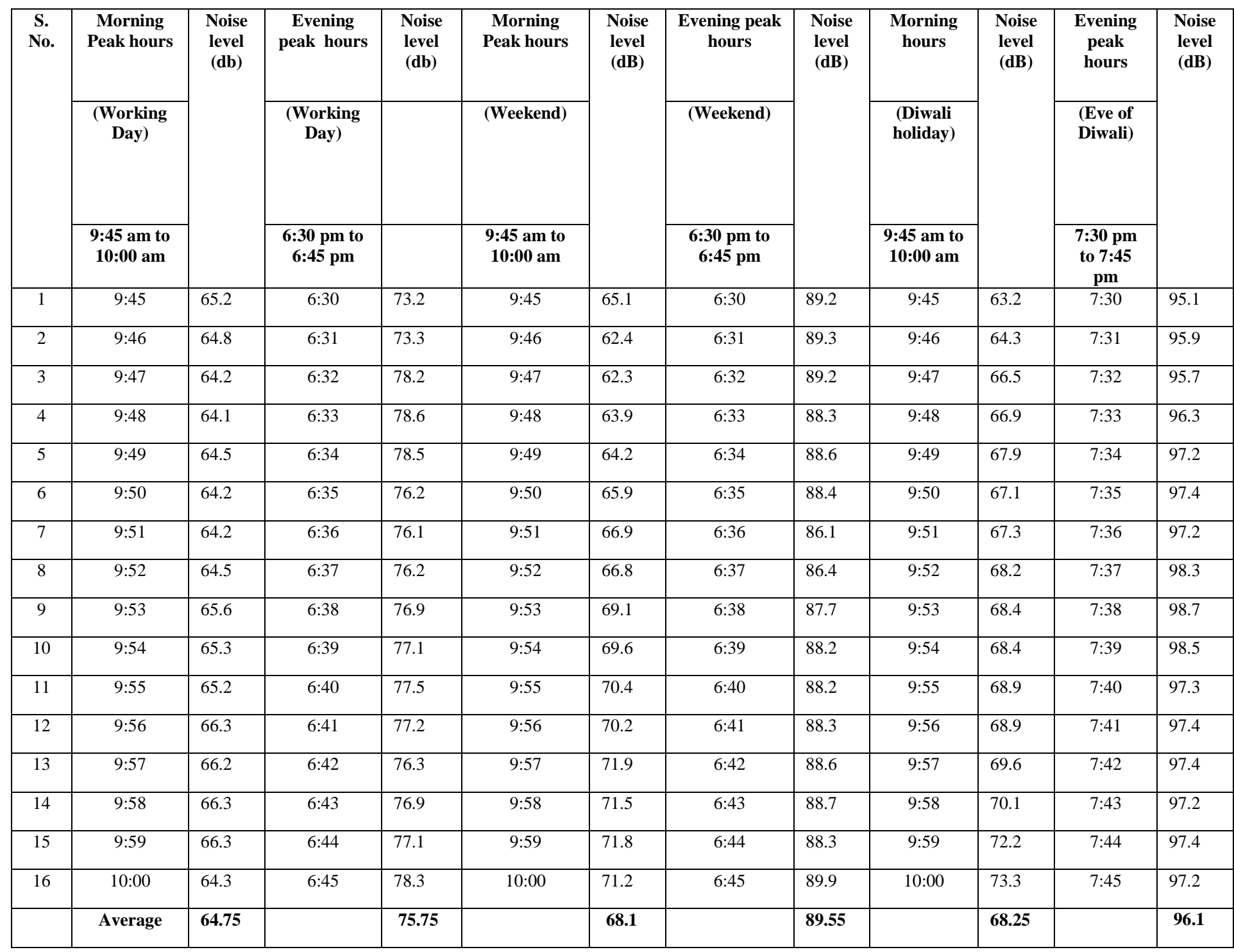


International Journal of Engineering Applied Sciences and Technology, 2019

Vol. 4, Issue 3, ISSN No. 2455-2143, Pages 573-605

Published Online July 2019 in IJEAST (http://www.ijeast.com)

Table 4: Noise level data at Afzal Gunj

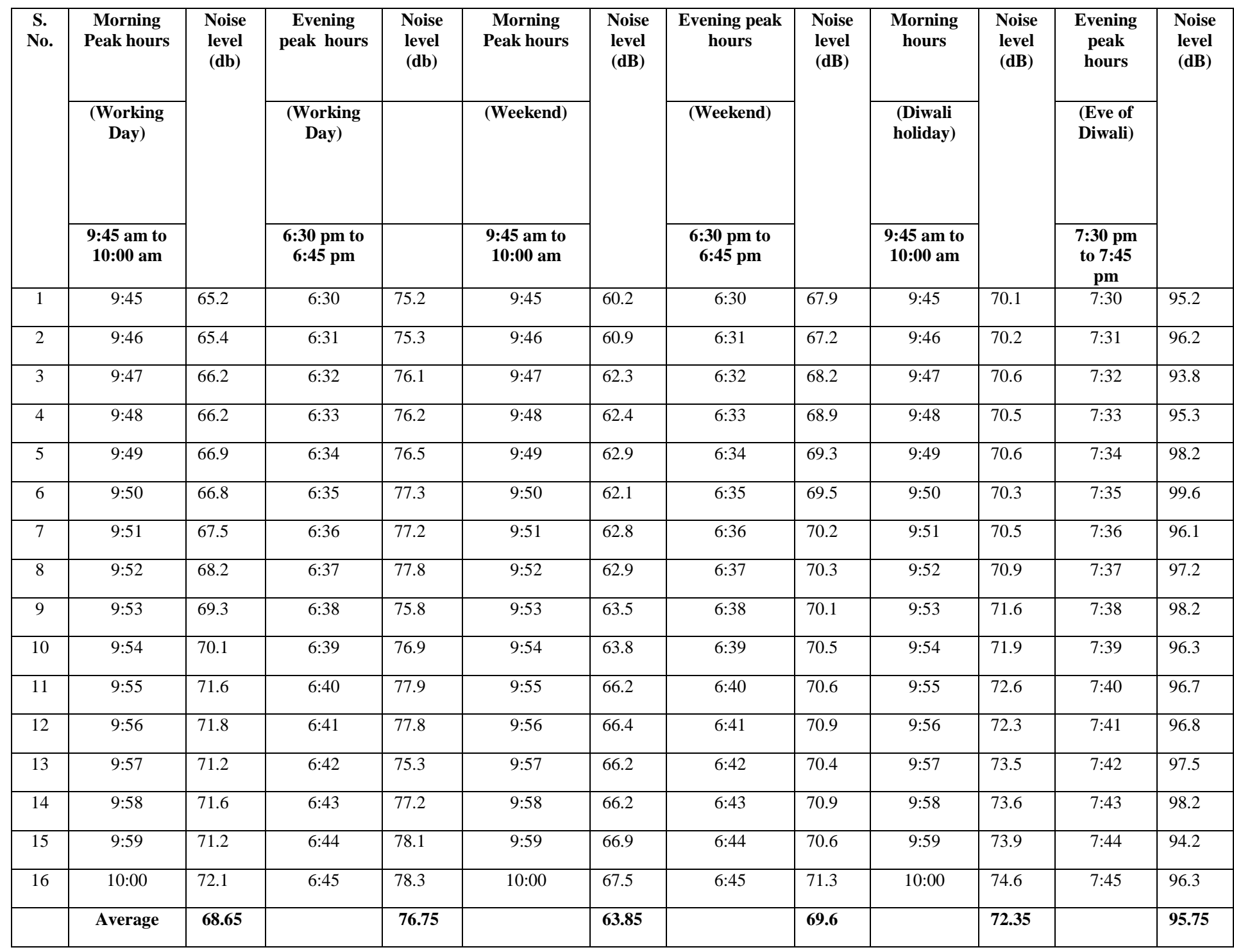


Published Online July 2019 in IJEAST (http://www.ijeast.com)

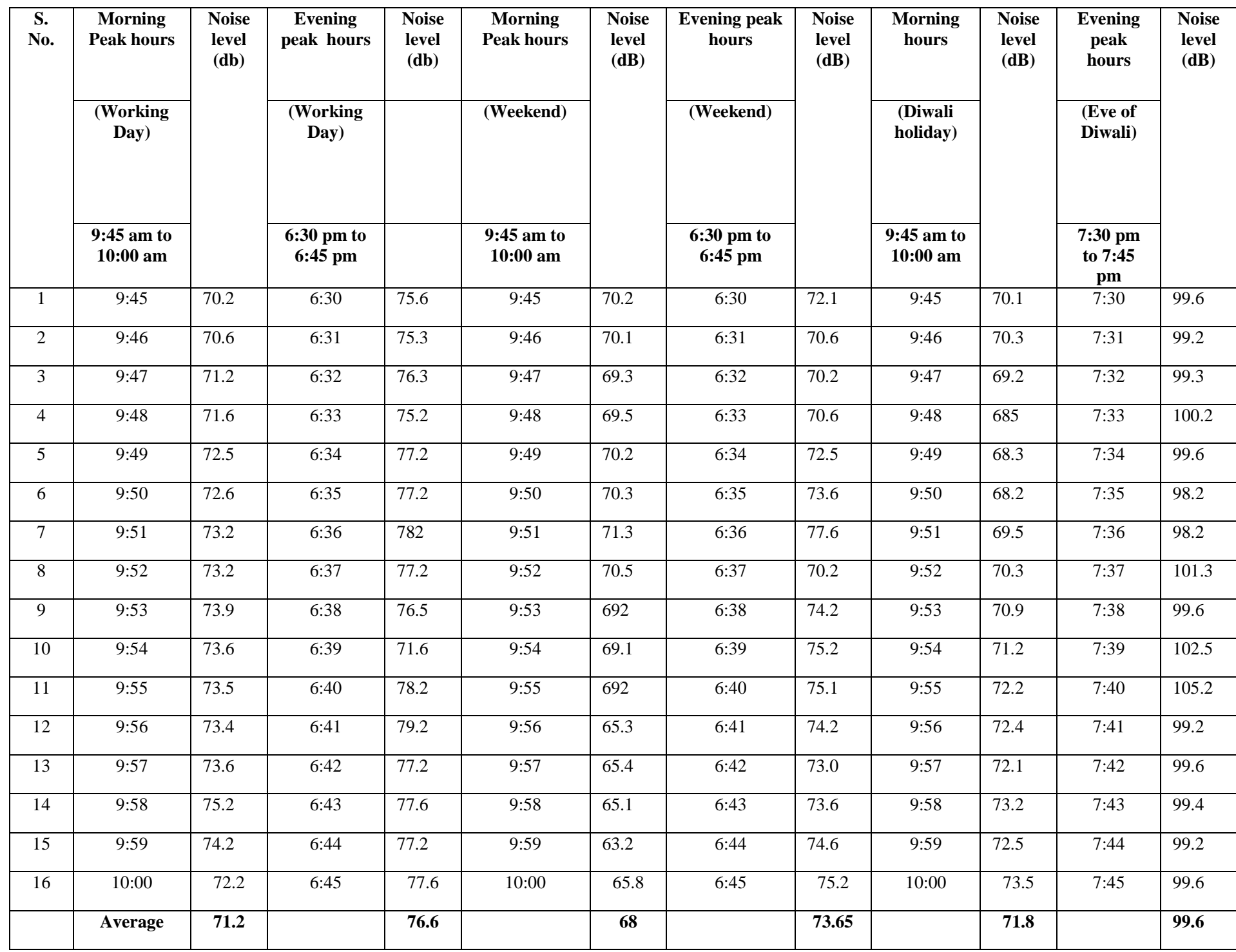

Table 5: Noise level data at Mir alam Mandi 
Published Online July 2019 in IJEAST (http://www.ijeast.com)

\begin{tabular}{|c|c|c|c|c|c|c|c|c|c|c|c|c|}
\hline \multirow[t]{3}{*}{$\begin{array}{l}\text { S. } \\
\text { No. }\end{array}$} & $\begin{array}{c}\text { Morning } \\
\text { Peak } \\
\text { hours }\end{array}$ & \multirow[t]{3}{*}{$\begin{array}{l}\text { Noise } \\
\text { level } \\
\text { (db) }\end{array}$} & $\begin{array}{c}\text { Evening } \\
\text { peak } \\
\text { hours }\end{array}$ & $\begin{array}{l}\text { Noise } \\
\text { level } \\
\text { (db) }\end{array}$ & $\begin{array}{c}\text { Morning } \\
\text { Peak } \\
\text { hours }\end{array}$ & \multirow[t]{3}{*}{$\begin{array}{l}\text { Noise } \\
\text { level } \\
\text { (dB) }\end{array}$} & $\begin{array}{c}\text { Evening } \\
\text { peak } \\
\text { hours }\end{array}$ & \multirow[t]{3}{*}{$\begin{array}{l}\text { Noise } \\
\text { level } \\
\text { (dB) }\end{array}$} & $\begin{array}{c}\text { Morning } \\
\text { hours }\end{array}$ & \multirow[t]{3}{*}{$\begin{array}{l}\text { Noise } \\
\text { level } \\
\text { (dB) }\end{array}$} & $\begin{array}{c}\text { Evening } \\
\text { peak } \\
\text { hours }\end{array}$ & \multirow[t]{3}{*}{$\begin{array}{l}\text { Noise } \\
\text { level } \\
\text { (dB) }\end{array}$} \\
\hline & $\begin{array}{c}\text { (Working } \\
\text { Day) }\end{array}$ & & $\begin{array}{c}\text { (Working } \\
\text { Day) }\end{array}$ & & (Weekend) & & (Weekend) & & $\begin{array}{c}\text { (Diwali } \\
\text { holiday) }\end{array}$ & & $\begin{array}{l}\text { (Eve of } \\
\text { Diwali) }\end{array}$ & \\
\hline & $\begin{array}{l}\text { 9:45 am } \\
\text { to } 10: 00 \\
\text { am }\end{array}$ & & $\begin{array}{c}6: 30 \mathrm{pm} \\
\text { to } 6: 45 \\
\text { pm }\end{array}$ & & $\begin{array}{c}\text { 9:45 am to } \\
10: 00 \text { am }\end{array}$ & & $\begin{array}{c}6: 30 \mathrm{pm} \text { to } \\
6: 45 \mathrm{pm}\end{array}$ & & $\begin{array}{c}\text { 9:45 am } \\
\text { to } 10: 00 \\
\text { am }\end{array}$ & & $\begin{array}{c}7: 30 \mathrm{pm} \\
\text { to } 7: 45 \\
\text { pm }\end{array}$ & \\
\hline 1 & $9: 45$ & 71.3 & $6: 30$ & 76.5 & $9: 45$ & 65.3 & $6: 30$ & 70.2 & $9: 45$ & 65.3 & $7: 30$ & 99.3 \\
\hline 2 & $9: 46$ & 71.2 & $6: 31$ & 77.5 & $9: 46$ & 62.3 & $6: 31$ & 71.3 & $9: 46$ & 65.2 & $7: 31$ & 99.6 \\
\hline 3 & $9: 47$ & 70.6 & $6: 32$ & 779 & $9: 47$ & 62.4 & $6: 32$ & 74.6 & $9: 47$ & 66.2 & $7: 32$ & 99.5 \\
\hline 4 & $9: 48$ & 70.2 & $6: 33$ & 78.6 & $9: 48$ & 62.4 & $6: 33$ & 74.2 & $9: 48$ & 66.2 & $7: 33$ & 99.4 \\
\hline 5 & $9: 49$ & 70.6 & $6: 34$ & 78.9 & $9: 49$ & 63.7 & $6: 34$ & 74.9 & $9: 49$ & 66.8 & $7: 34$ & 99.6 \\
\hline 6 & $9: 50$ & 70.5 & $6: 35$ & 79.5 & $9: 50$ & 64.2 & $6: 35$ & 75.6 & $9: 50$ & 67.5 & $7: 35$ & 98.5 \\
\hline 7 & $9: 51$ & 70.3 & $6: 36$ & 79.6 & $9: 51$ & 65.2 & $6: 36$ & 75.2 & $9: 51$ & 67.4 & $7: 36$ & 96.5 \\
\hline 8 & $9: 52$ & 70.1 & $6: 37$ & 79.5 & $9: 52$ & 651 & $6: 37$ & 75.9 & $9: 52$ & 67.3 & $7: 37$ & 97.2 \\
\hline 9 & $9: 53$ & 69.2 & $6: 38$ & 80.1 & $9: 53$ & 66.5 & $6: 38$ & 74.2 & $9: 53$ & 67.6 & $7: 38$ & 95.2 \\
\hline 10 & $9: 54$ & 69.5 & $6: 39$ & 80.2 & $9: 54$ & 66.8 & $6: 39$ & 74.2 & $9: 54$ & 67.4 & $7: 39$ & 96.2 \\
\hline 11 & $9: 55$ & 68.5 & $6: 40$ & 82.3 & $9: 55$ & 66.7 & $6: 40$ & 74.3 & $9: 55$ & 67.2 & $7: 40$ & 98.5 \\
\hline 12 & $9: 56$ & 70.6 & $6: 41$ & 82.3 & $9: 56$ & 67.1 & $6: 41$ & 76.3 & $9: 56$ & 67.2 & $7: 41$ & 99.3 \\
\hline 13 & $9: 57$ & 69.2 & $6: 42$ & 81.6 & $9: 57$ & 67.2 & $6: 42$ & 75.3 & 9:57 & 68.2 & $7: 42$ & 99.2 \\
\hline 14 & $9: 58$ & 70.2 & $6: 43$ & 81.2 & $9: 58$ & 67.2 & $6: 43$ & 74.2 & $9: 58$ & 67.2 & $7: 43$ & 99.6 \\
\hline 15 & $9: 59$ & 71.2 & $6: 44$ & 82.2 & $9: 59$ & 66.9 & $6: 44$ & 72.5 & $9: 59$ & 66.2 & $7: 44$ & 100.1 \\
\hline \multirow[t]{2}{*}{16} & 10:00 & 70.2 & $6: 45$ & 83.1 & 10:00 & 66.4 & $6: 45$ & 74.2 & 10:00 & 64.2 & $7: 45$ & 99.9 \\
\hline & Average & 70.75 & & 79.8 & & 65.85 & & 72.2 & & 64.75 & & 99.3 \\
\hline
\end{tabular}

Table 6: Noise level data at Begum Bazaar 
Published Online July 2019 in IJEAST (http://www.ijeast.com)

Table 7: Noise level data at Sultan Bazaar

\begin{tabular}{|c|c|c|c|c|c|c|c|c|c|c|c|c|}
\hline \multirow[t]{3}{*}{$\begin{array}{c}\text { S. } \\
\text { No. }\end{array}$} & $\begin{array}{c}\text { Morning } \\
\text { Peak hours }\end{array}$ & \multirow[t]{3}{*}{$\begin{array}{c}\text { Noise } \\
\text { level } \\
\text { (db) }\end{array}$} & $\begin{array}{c}\text { Evening } \\
\text { peak hours }\end{array}$ & $\begin{array}{c}\text { Noise } \\
\text { level } \\
(d b)\end{array}$ & $\begin{array}{c}\text { Morning } \\
\text { Peak hours }\end{array}$ & \multirow[t]{3}{*}{$\begin{array}{l}\text { Noise } \\
\text { level } \\
\text { (dB) }\end{array}$} & $\begin{array}{c}\text { Evening peak } \\
\text { hours }\end{array}$ & \multirow[t]{3}{*}{$\begin{array}{c}\text { Noise } \\
\text { level } \\
\text { (dB) }\end{array}$} & $\begin{array}{c}\text { Morning } \\
\text { hours }\end{array}$ & \multirow[t]{3}{*}{$\begin{array}{c}\text { Noise } \\
\text { level } \\
\text { (dB) }\end{array}$} & $\begin{array}{c}\text { Evening } \\
\text { peak } \\
\text { hours }\end{array}$ & \multirow[t]{3}{*}{$\begin{array}{l}\text { Noise } \\
\text { level } \\
\text { (dB) }\end{array}$} \\
\hline & $\begin{array}{c}\text { (Working } \\
\text { Day) }\end{array}$ & & $\begin{array}{c}\text { (Working } \\
\text { Day) }\end{array}$ & & (Weekend) & & (Weekend) & & $\begin{array}{c}\text { (Diwali } \\
\text { holiday) }\end{array}$ & & $\begin{array}{l}\text { (Eve of } \\
\text { Diwali) }\end{array}$ & \\
\hline & $\begin{array}{l}9: 45 \text { am to } \\
10: 00 \text { am }\end{array}$ & & $\begin{array}{c}6: 30 \mathrm{pm} \text { to } \\
6: 45 \mathrm{pm}\end{array}$ & & $\begin{array}{c}\text { 9:45 am to } \\
10: 00 \mathrm{am}\end{array}$ & & $\begin{array}{c}6: 30 \mathrm{pm} \text { to } \\
6: 45 \mathrm{pm}\end{array}$ & & $\begin{array}{c}\text { 9:45 am to } \\
10: 00 \text { am }\end{array}$ & & $\begin{array}{c}7: 30 \mathrm{pm} \\
\text { to } 7: 45 \\
\text { pm }\end{array}$ & \\
\hline 1 & $9: 45$ & 72.2 & $6: 30$ & 75.2 & $9: 45$ & 65.6 & $6: 30$ & 63.5 & $9: 45$ & 64.2 & $7: 30$ & 97.2 \\
\hline 2 & $9: 46$ & 72.3 & $6: 31$ & 7.6 & $9: 46$ & 653 & $6: 31$ & 63.5 & $9: 46$ & 64.2 & $7: 31$ & 97.4 \\
\hline 3 & $9: 47$ & 72.4 & $6: 32$ & 75.9 & $9: 47$ & 652 & $6: 32$ & 63.2 & $9: 47$ & 63.2 & $7: 32$ & 97.6 \\
\hline 4 & $9: 48$ & 72.9 & $6: 33$ & 76.2 & $9: 48$ & 66.1 & $6: 33$ & 63.8 & $9: 48$ & 64.2 & $7: 33$ & 97.5 \\
\hline 5 & $9: 49$ & 73.5 & $6: 34$ & 76.9 & $9: 49$ & 66.2 & $6: 34$ & 64.2 & $9: 49$ & 64.3 & $7: 34$ & 97.8 \\
\hline 6 & $9: 50$ & 73.6 & $6: 35$ & 77.5 & $9: 50$ & 66.7 & $6: 35$ & 64.2 & $9: 50$ & 64.5 & $7: 35$ & 98.5 \\
\hline 7 & $9: 51$ & 73.5 & $6: 36$ & 77.2 & $9: 51$ & 66.8 & $6: 36$ & 64.5 & $9: 51$ & 64.9 & $7: 36$ & 98.1 \\
\hline 8 & $9: 52$ & 74.2 & $6: 37$ & 77.6 & $9: 52$ & 66.2 & $6: 37$ & 64.3 & $9: 52$ & 65.2 & $7: 37$ & 98.2 \\
\hline 9 & $9: 53$ & 74.2 & $6: 38$ & 77.2 & $9: 53$ & 66.1 & $6: 38$ & 64.9 & $9: 53$ & 65.2 & $7: 38$ & 98.3 \\
\hline 10 & $9: 54$ & 74.5 & $6: 39$ & 78.6 & $9: 54$ & 66.3 & $6: 39$ & 65.1 & $9: 54$ & 65.3 & $7: 39$ & 98.5 \\
\hline 11 & $9: 55$ & 72.3 & $6: 40$ & 77.5 & $9: 55$ & 67.1 & $6: 40$ & 65.1 & $9: 55$ & 65.7 & $7: 40$ & 98.4 \\
\hline 12 & $9: 56$ & 72.3 & $6: 41$ & 77.9 & $9: 56$ & 67.1 & $6: 41$ & 65.2 & $9: 56$ & 65.9 & $7: 41$ & 98.7 \\
\hline 13 & $9: 57$ & 72.3 & $6: 42$ & 77.6 & $9: 57$ & 66.9 & $6: 42$ & 65.5 & $9: 57$ & 65.3 & $7: 42$ & 98.4 \\
\hline 14 & $9: 58$ & 73.2 & $6: 43$ & 77.4 & $9: 58$ & 66.4 & $6: 43$ & 65.6 & 9:58 & 65.2 & $7: 43$ & 98.6 \\
\hline 15 & $9: 59$ & 73.5 & $6: 44$ & 77.2 & $9: 59$ & 66.1 & $6: 44$ & 65.2 & $9: 59$ & 65.8 & $7: 44$ & 98.4 \\
\hline 16 & $10: 00$ & 73.4 & $6: 45$ & 77.1 & 10:00 & 66.2 & $6: 45$ & 65.8 & $10: 00$ & 65.9 & $7: 45$ & 98.2 \\
\hline & Average & 72.8 & & 76.15 & & 65.9 & & 64.65 & & 65.05 & & 97.7 \\
\hline
\end{tabular}


Published Online July 2019 in IJEAST (http://www.ijeast.com)

Table 8: Noise level data at Kothi

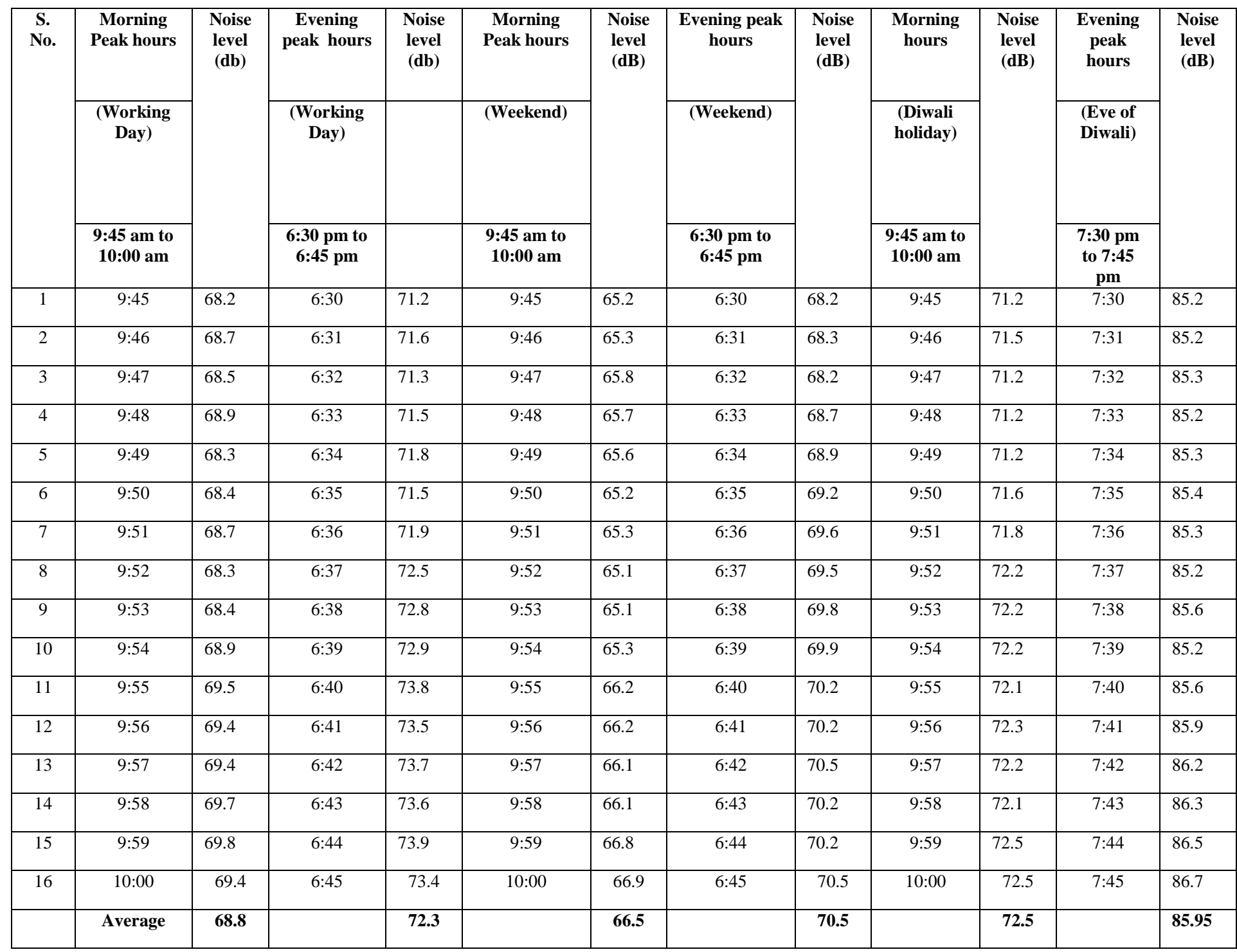


Published Online July 2019 in IJEAST (http://www.ijeast.com)

Table 9: Noise level data at Abids

\begin{tabular}{|c|c|c|c|c|c|c|c|c|c|c|c|c|}
\hline \multirow[t]{3}{*}{$\begin{array}{l}\text { S. } \\
\text { No. }\end{array}$} & $\begin{array}{c}\text { Morning } \\
\text { Peak hours }\end{array}$ & \multirow[t]{3}{*}{$\begin{array}{l}\text { Noise } \\
\text { level } \\
\text { (db) }\end{array}$} & $\begin{array}{c}\text { Evening } \\
\text { peak hours }\end{array}$ & $\begin{array}{l}\text { Noise } \\
\text { level } \\
\text { (db) }\end{array}$ & $\begin{array}{c}\text { Morning } \\
\text { Peak hours }\end{array}$ & \multirow[t]{3}{*}{$\begin{array}{l}\text { Noise } \\
\text { level } \\
\text { (dB) }\end{array}$} & $\begin{array}{c}\text { Evening peak } \\
\text { hours }\end{array}$ & \multirow[t]{3}{*}{$\begin{array}{l}\text { Noise } \\
\text { level } \\
\text { (dB) }\end{array}$} & $\begin{array}{c}\text { Morning } \\
\text { hours }\end{array}$ & \multirow[t]{3}{*}{$\begin{array}{l}\text { Noise } \\
\text { level } \\
\text { (dB) }\end{array}$} & $\begin{array}{c}\text { Evening } \\
\text { peak } \\
\text { hours }\end{array}$ & \multirow[t]{3}{*}{$\begin{array}{l}\text { Noise } \\
\text { level } \\
\text { (dB) }\end{array}$} \\
\hline & $\begin{array}{c}\text { (Working } \\
\text { Day) }\end{array}$ & & $\begin{array}{l}\text { (Working } \\
\text { Day) }\end{array}$ & & (Weekend) & & (Weekend) & & $\begin{array}{c}\text { (Diwali } \\
\text { holiday) }\end{array}$ & & $\begin{array}{l}\text { (Eve of } \\
\text { Diwali) }\end{array}$ & \\
\hline & $\begin{array}{l}\text { 9:45 am to } \\
10: 00 \text { am }\end{array}$ & & $\begin{array}{c}\text { 6:30 pm to } \\
6: 45 \mathrm{pm}\end{array}$ & & $\begin{array}{c}\text { 9:45 am to } \\
10: 00 \text { am }\end{array}$ & & $\begin{array}{c}\text { 6:30 pm to } \\
6: 45 \mathrm{pm}\end{array}$ & & $\begin{array}{l}\text { 9:45 am to } \\
10: 00 \text { am }\end{array}$ & & $\begin{array}{c}7: 30 \mathrm{pm} \\
\text { to } 7: 45 \\
\text { pm }\end{array}$ & \\
\hline 1 & $9: 45$ & 69.2 & $6: 30$ & 72.5 & $9: 45$ & 65.2 & $6: 30$ & 72.2 & $9: 45$ & 75.2 & 7:30 & 96.2 \\
\hline 2 & $9: 46$ & 69.3 & $6: 31$ & 72.6 & 9:46 & 65.2 & $6: 31$ & 72.2 & $9: 46$ & 75.2 & $7: 31$ & 96.2 \\
\hline 3 & 9:47 & 69.6 & $6: 32$ & 72.5 & 9:47 & 65.2 & $6: 32$ & 72.3 & $9: 47$ & 75.3 & 7:32 & 96.2 \\
\hline 4 & $9: 48$ & 70.2 & $6: 33$ & 72.5 & 9:48 & 65.3 & $6: 33$ & 72.3 & $9: 48$ & 75.3 & $7: 33$ & 96.3 \\
\hline 5 & $9: 49$ & 70.2 & $6: 34$ & 73.5 & 9:49 & 65.6 & $6: 34$ & 72.4 & 9:49 & 75.2 & $7: 34$ & 96.1 \\
\hline 6 & $9: 50$ & 70.6 & $6: 35$ & 73.6 & 9:50 & 65.6 & $6: 35$ & 72.6 & $9: 50$ & 75.2 & $7: 35$ & 96.1 \\
\hline 7 & $9: 51$ & 70.2 & $6: 36$ & 73.6 & 9:51 & 65.7 & $6: 36$ & 72.6 & 9:51 & 75.2 & 7:36 & 97.2 \\
\hline 8 & $9: 52$ & 70.2 & $6: 37$ & 73.1 & $9: 52$ & 65.9 & $6: 37$ & 72.8 & $9: 52$ & 75.3 & $7: 37$ & 97.2 \\
\hline 9 & $9: 53$ & 70.2 & $6: 38$ & 73.8 & $9: 53$ & 63.8 & $6: 38$ & 73.1 & $9: 53$ & 76.2 & $7: 38$ & 97.5 \\
\hline 10 & 9:54 & 71.3 & 6:39 & 73.5 & 9:54 & 63.2 & 6:39 & 73.2 & 9:54 & 76.3 & 7:39 & 97.5 \\
\hline 11 & $9: 55$ & 71.3 & $6: 40$ & 73.5 & $9: 55$ & 63.1 & $6: 40$ & 73.2 & $9: 55$ & 76.3 & $7: 40$ & 97.5 \\
\hline 12 & $9: 56$ & 71.3 & $6: 41$ & 73.5 & $9: 56$ & 63.6 & $6: 41$ & 734 & $9: 56$ & 76.2 & 7:41 & 97.8 \\
\hline 13 & $9: 57$ & 71.5 & $6: 42$ & 73.6 & 9:57 & 63.8 & $6: 42$ & 73.4 & 9:57 & 76.2 & $7: 42$ & 97.8 \\
\hline 14 & 9:58 & 71.6 & $6: 43$ & 73.8 & 9:58 & 63.7 & $6: 43$ & 73.4 & 9:58 & 76.2 & 7:43 & 97.8 \\
\hline 15 & $9: 59$ & 71.5 & $6: 44$ & 73.6 & 9:59 & 63.8 & $6: 44$ & 73.6 & $9: 59$ & 76.2 & $7: 44$ & 97.8 \\
\hline 16 & 10:00 & 71.6 & $6: 45$ & 73.4 & 10:00 & 63.2 & $6: 45$ & 73.8 & 10:00 & 76.2 & 7:45 & 96.8 \\
\hline & Average & 70.4 & & 72.95 & & 64.2 & & 73 & & 75.7 & & 96.5 \\
\hline
\end{tabular}


Published Online July 2019 in IJEAST (http://www.ijeast.com)

Table 10: Noise level data at Mehdipatnam

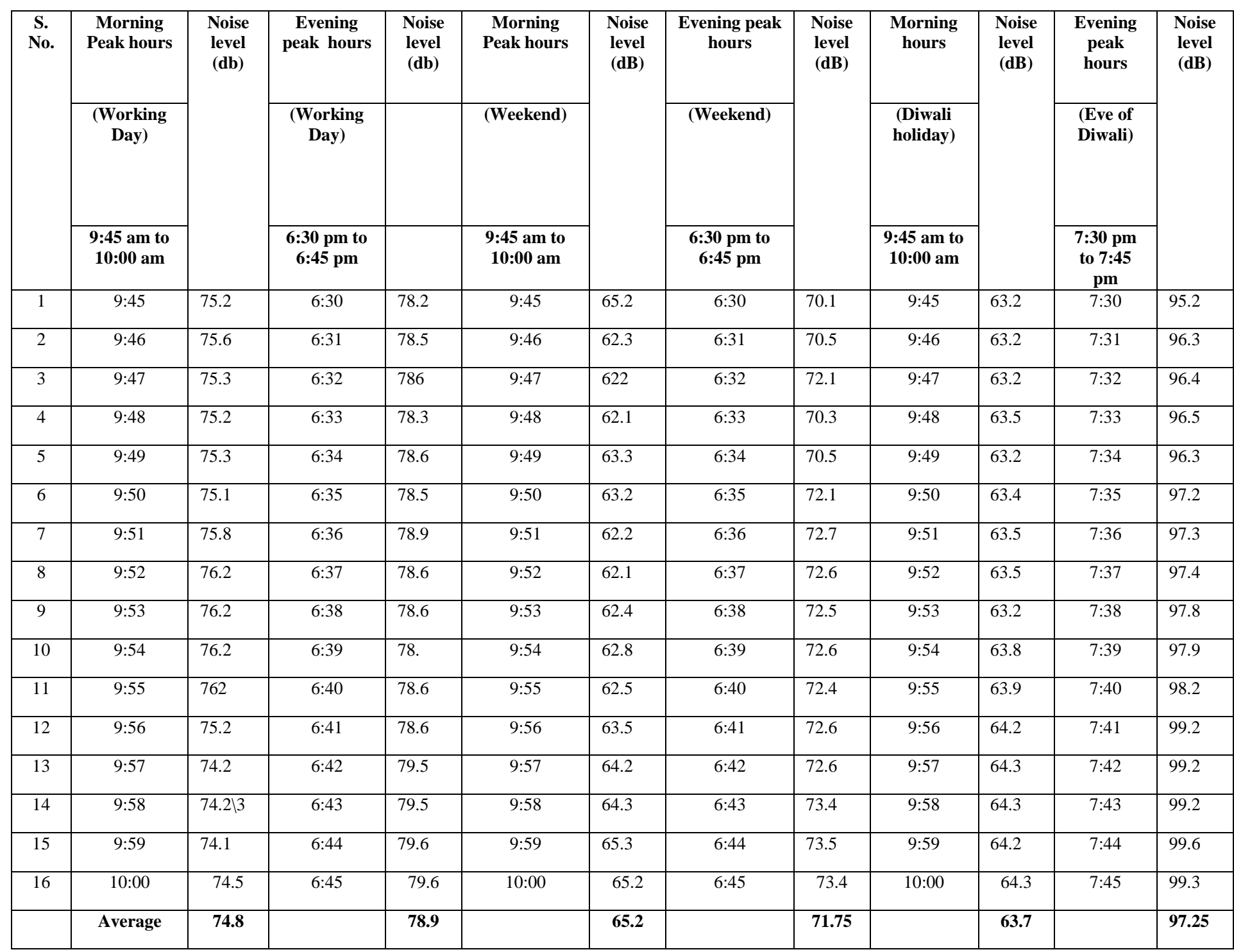


Published Online July 2019 in IJEAST (http://www.ijeast.com)

\begin{tabular}{|c|c|c|c|c|c|c|c|c|c|c|c|c|}
\hline \multirow[t]{3}{*}{$\begin{array}{l}\text { S. } \\
\text { No. }\end{array}$} & $\begin{array}{c}\text { Morning } \\
\text { Peak } \\
\text { hours }\end{array}$ & \multirow[t]{3}{*}{$\begin{array}{l}\text { Noise } \\
\text { level } \\
\text { (db) }\end{array}$} & $\begin{array}{c}\text { Evening } \\
\text { peak } \\
\text { hours }\end{array}$ & $\begin{array}{l}\text { Noise } \\
\text { level } \\
\text { (db) }\end{array}$ & $\begin{array}{c}\text { Morning } \\
\text { Peak } \\
\text { hours }\end{array}$ & \multirow[t]{3}{*}{$\begin{array}{l}\text { Noise } \\
\text { level } \\
\text { (dB) }\end{array}$} & $\begin{array}{c}\text { Evening } \\
\text { peak } \\
\text { hours }\end{array}$ & \multirow[t]{3}{*}{$\begin{array}{l}\text { Noise } \\
\text { level } \\
\text { (dB) }\end{array}$} & $\begin{array}{c}\text { Morning } \\
\text { hours }\end{array}$ & \multirow[t]{3}{*}{$\begin{array}{l}\text { Noise } \\
\text { level } \\
\text { (dB) }\end{array}$} & $\begin{array}{c}\text { Evening } \\
\text { peak } \\
\text { hours }\end{array}$ & \multirow[t]{3}{*}{$\begin{array}{l}\text { Noise } \\
\text { level } \\
\text { (dB) }\end{array}$} \\
\hline & $\begin{array}{c}\text { (Working } \\
\text { Day) }\end{array}$ & & $\begin{array}{c}\text { (Working } \\
\text { Day) }\end{array}$ & & (Weekend) & & (Weekend) & & $\begin{array}{c}\text { (Diwali } \\
\text { holiday) }\end{array}$ & & $\begin{array}{l}\text { (Eve of } \\
\text { Diwali) }\end{array}$ & \\
\hline & $\begin{array}{c}9: 45 \text { am } \\
\text { to } 10: 00 \\
\text { am } \\
\end{array}$ & & $\begin{array}{c}6: 30 \mathrm{pm} \\
\text { to } 6: 45 \\
\mathrm{pm} \\
\end{array}$ & & $\begin{array}{l}\text { 9:45 am to } \\
\text { 10:00 am }\end{array}$ & & $\begin{array}{c}6: 30 \mathrm{pm} \text { to } \\
6: 45 \mathrm{pm}\end{array}$ & & $\begin{array}{c}9: 45 \text { am } \\
\text { to } 10: 00 \\
\text { am } \\
\end{array}$ & & $\begin{array}{c}7: 30 \mathrm{pm} \\
\text { to } 7: 45 \\
\text { pm } \\
\end{array}$ & \\
\hline 1 & 9:45 & 65.2 & $6: 30$ & 69.3 & $9: 45$ & 60.2 & $6: 30$ & 72.5 & $9: 45$ & 63.2 & $7: 30$ & 98.6 \\
\hline 2 & $9: 46$ & 65.3 & $6: 31$ & 69.3 & 9:46 & 60.1 & $6: 31$ & 72.5 & $9: 46$ & 63.2 & $7: 31$ & 98.6 \\
\hline 3 & $9: 47$ & 65.3 & $6: 32$ & 69.2 & $9: 47$ & 60.2 & $6: 32$ & 72.5 & 9:47 & 63.2 & $7: 32$ & 99.6 \\
\hline 4 & 9:48 & 65.3 & $6: 33$ & 69.2 & 9:48 & 60.2 & $6: 33$ & 72.5 & $9: 48$ & 63.5 & $7: 33$ & 100.1 \\
\hline 5 & 9:49 & 65.2 & $6: 34$ & 69.2 & 9:49 & 60.2 & $6: 34$ & 72.6 & 9:49 & 63.2 & $7: 34$ & 100.1 \\
\hline 6 & $9: 50$ & 65.4 & $6: 35$ & 69.2 & 9:50 & 60.5 & $6: 35$ & 72.6 & $9: 50$ & 63.5 & $7: 35$ & 100.2 \\
\hline 7 & $9: 51$ & 65.2 & $6: 36$ & 69.3 & $9: 51$ & 61.2 & $6: 36$ & 72.6 & $9: 51$ & 63.5 & $7: 36$ & 100.2 \\
\hline 8 & $9: 52$ & 65.3 & 6:37 & 70.1 & $9: 52$ & 61.2 & 6:37 & 72.7 & $9: 52$ & 63.8 & $7: 37$ & 99.6 \\
\hline 9 & $9: 53$ & 65.4 & $6: 38$ & 70.2 & $9: 53$ & 61.2 & $6: 38$ & 72.8 & $9: 53$ & 64.2 & $7: 38$ & 99.8 \\
\hline 10 & 9:54 & 65.3 & $6: 39$ & 70.1 & 9:54 & 60.2 & $6: 39$ & 72.9 & $9: 54$ & 64.4 & $7: 39$ & 99.8 \\
\hline 11 & $9: 55$ & 65.2 & $6: 40$ & 70.1 & $9: 55$ & 60.2 & $6: 40$ & 72.8 & $9: 55$ & 64.2 & $7: 40$ & 99.8 \\
\hline 12 & $9: 56$ & 65.3 & $6: 41$ & 70.3 & $9: 56$ & 60.2 & $6: 41$ & 72.9 & $9: 56$ & 65.3 & $7: 41$ & 99.7 \\
\hline 13 & 9:57 & 65.8 & $6: 42$ & 70.3 & 9:57 & 60.2 & $6: 42$ & 73.5 & 9:57 & 65.3 & $7: 42$ & 99.3 \\
\hline 14 & $9: 58$ & 65.9 & $6: 43$ & 70.4 & $9: 58$ & 60.3 & $6: 43$ & 73.5 & $9: 58$ & 65.3 & $7: 43$ & 99.2 \\
\hline 15 & $9: 59$ & 65.9 & $6: 44$ & 70.5 & $9: 59$ & 60.3 & $6: 44$ & 73.5 & $9: 59$ & 65.3 & $7: 44$ & 99.7 \\
\hline 16 & $10: 00$ & 66.1 & $6: 45$ & 70.5 & 10:00 & 60.5 & $6: 45$ & 73.6 & $10: 00$ & 65.2 & $7: 45$ & 99.6 \\
\hline & Average & 65.65 & & 69.9 & & 60.35 & & 73.05 & & 64.2 & & 99.1 \\
\hline
\end{tabular}

Table 11: Noise level data at Nampally 
Published Online July 2019 in IJEAST (http://www.ijeast.com)

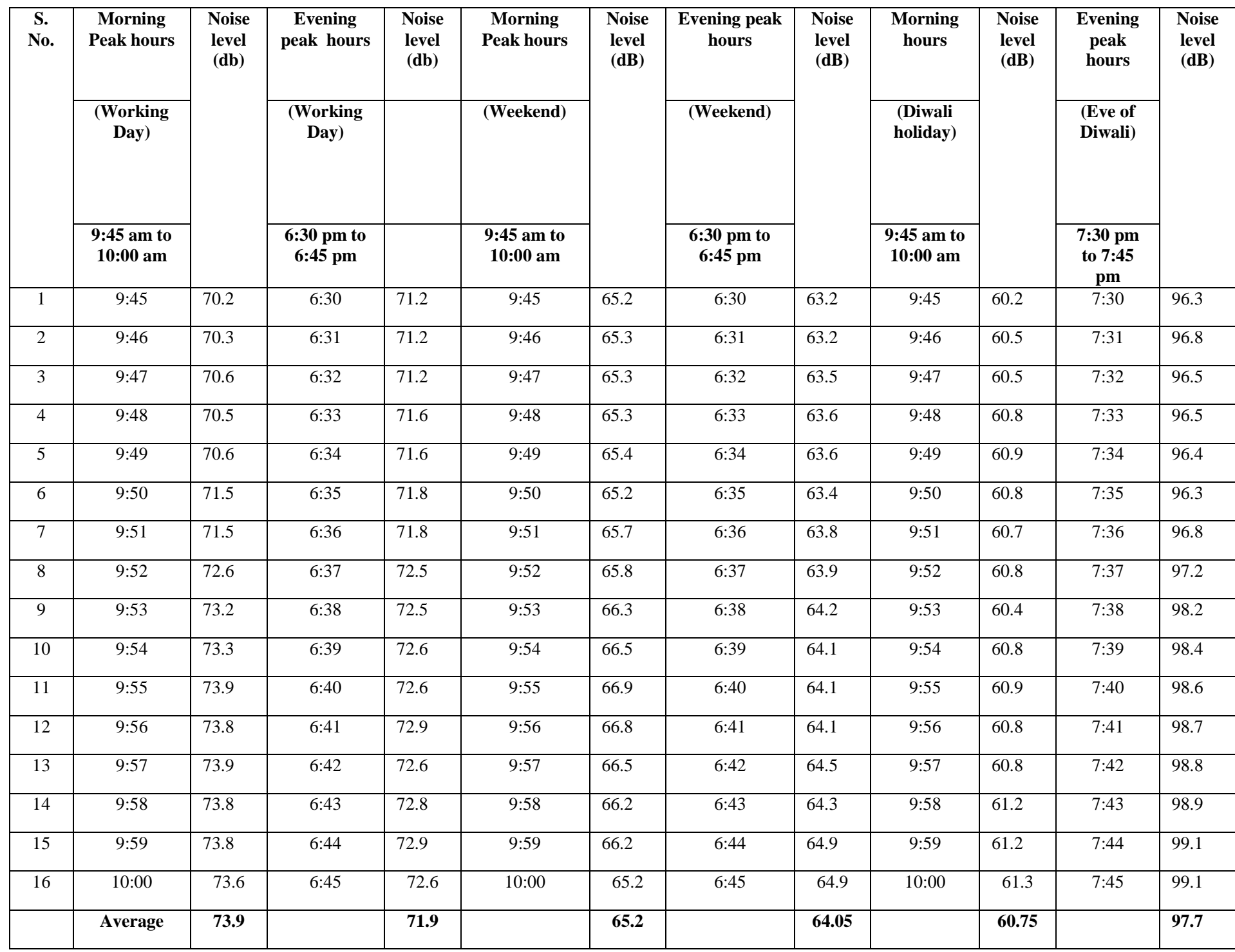

Table 12: Noise level data at Lakdi- ka-pool 
Published Online July 2019 in IJEAST (http://www.ijeast.com)

\begin{tabular}{|c|c|c|c|c|c|c|c|c|c|c|c|c|}
\hline \multirow[t]{3}{*}{$\begin{array}{l}\text { S. } \\
\text { No. }\end{array}$} & $\begin{array}{c}\text { Morning } \\
\text { Peak hours }\end{array}$ & $\begin{array}{l}\text { Noise } \\
\text { level } \\
\text { (db) }\end{array}$ & $\begin{array}{c}\text { Evening } \\
\text { peak hours }\end{array}$ & $\begin{array}{l}\text { Noise } \\
\text { level } \\
\text { (db) }\end{array}$ & $\begin{array}{c}\text { Morning } \\
\text { Peak hours }\end{array}$ & \multirow[t]{3}{*}{$\begin{array}{l}\text { Noise } \\
\text { level } \\
\text { (dB) }\end{array}$} & $\begin{array}{l}\text { Evening peak } \\
\text { hours }\end{array}$ & \multirow[t]{3}{*}{$\begin{array}{l}\text { Noise } \\
\text { level } \\
\text { (dB) }\end{array}$} & $\begin{array}{c}\text { Morning } \\
\text { hours }\end{array}$ & \multirow[t]{3}{*}{$\begin{array}{l}\text { Noise } \\
\text { level } \\
\text { (dB) }\end{array}$} & $\begin{array}{c}\text { Evening } \\
\text { peak } \\
\text { hours }\end{array}$ & \multirow[t]{3}{*}{$\begin{array}{l}\text { Noise } \\
\text { level } \\
\text { (dB) }\end{array}$} \\
\hline & $\begin{array}{c}\text { (Working } \\
\text { Day) }\end{array}$ & & $\begin{array}{c}\text { (Working } \\
\text { Day) }\end{array}$ & & (Weekend) & & (Weekend) & & $\begin{array}{c}\text { (Diwali } \\
\text { holiday) }\end{array}$ & & $\begin{array}{l}\text { (Eve of } \\
\text { Diwali) }\end{array}$ & \\
\hline & $\begin{array}{c}\text { 9:45 am to } \\
10: 00 \text { am }\end{array}$ & & $\begin{array}{c}6: 30 \mathrm{pm} \text { to } \\
6: 45 \mathrm{pm}\end{array}$ & & $\begin{array}{c}\text { 9:45 am to } \\
10: 00 \text { am }\end{array}$ & & $\begin{array}{c}6: 30 \mathrm{pm} \text { to } \\
6: 45 \mathrm{pm}\end{array}$ & & $\begin{array}{l}\text { 9:45 am to } \\
10: 00 \mathrm{am}\end{array}$ & & $\begin{array}{c}7: 30 \mathrm{pm} \\
\text { to } 7: 45 \\
\text { pm }\end{array}$ & \\
\hline 1 & $9: 45$ & 74.5 & $6: 30$ & 75.6 & $9: 45$ & 65.2 & $6: 30$ & 66.2 & $9: 45$ & 65.3 & $7: 30$ & 96.3 \\
\hline 2 & 9:46 & 74.6 & $6: 31$ & 75.6 & $9: 46$ & 66.5 & $6: 31$ & 66.2 & $9: 46$ & 65.9 & $7: 31$ & 96.9 \\
\hline 3 & 9:47 & 74.8 & $6: 32$ & 758 & 9:47 & 66.8 & $6: 32$ & 663 & 9:47 & 65.8 & $7: 32$ & 97.6 \\
\hline 4 & 9:48 & 74.6 & $6: 33$ & 75.9 & 9:48 & 66.9 & $6: 33$ & 66.5 & 9:48 & 65.2 & $7: 33$ & 97.6 \\
\hline 5 & 9:49 & 74.9 & $6: 34$ & 76.6 & $9: 49$ & 67.5 & $6: 34$ & 66.3 & $9: 49$ & 65.4 & $7: 34$ & 97.6 \\
\hline 6 & $9: 50$ & 74.6 & $6: 35$ & 76.3 & $9: 50$ & 68.2 & $6: 35$ & 66.9 & $9: 50$ & 65.9 & $7: 35$ & 97.8 \\
\hline 7 & $9: 51$ & 74.9 & $6: 36$ & 76.6 & $9: 51$ & 68.2 & $6: 36$ & 668 & $9: 51$ & 65.8 & $7: 36$ & 97.9 \\
\hline 8 & $9: 52$ & 74.3 & $6: 37$ & 76.3 & $9: 52$ & 68.3 & $6: 37$ & 67.2 & $9: 52$ & 65.7 & $7: 37$ & 98.5 \\
\hline 9 & $9: 53$ & 74.9 & 6:38 & 76.2 & $9: 53$ & 68.3 & $6: 38$ & 67.3 & $9: 53$ & 65.8 & $7: 38$ & 98.5 \\
\hline 10 & $9: 54$ & 75.8 & $6: 39$ & 76.1 & $9: 54$ & 68.3 & $6: 39$ & 67.2 & $9: 54$ & 65.9 & $7: 39$ & 98.5 \\
\hline 11 & $9: 55$ & $\begin{array}{l}75.8 \\
\end{array}$ & $6: 40$ & 76.9 & $9: 55$ & 68.4 & $6: 40$ & 67.3 & $9: 55$ & 65.8 & $7: 40$ & 98.6 \\
\hline 12 & $9: 56$ & 75.9 & $6: 41$ & 77.5 & 9:56 & 69.4 & $6: 41$ & 67.4 & 9:56 & 65.9 & 7:41 & 99.5 \\
\hline 13 & 9:57 & 75.6 & $6: 42$ & 77.5 & 9:57 & 68.2 & $6: 42$ & 67.9 & 9:57 & 66.2 & 7:42 & 99.9 \\
\hline 14 & $9: 58$ & 75.9 & $6: 43$ & 76.5 & 9:58 & 68.2 & $6: 43$ & 68.2 & $9: 58$ & 66.3 & $7: 43$ & 99.8 \\
\hline 15 & $9: 59$ & 76.2 & $6: 44$ & 76.2 & 9:59 & 69.1 & $6: 44$ & 68.3 & $9: 59$ & 66.4 & $7: 44$ & 99.9 \\
\hline 16 & 10:00 & 76.6 & $6: 45$ & 76.3 & 10:00 & 69.2 & $6: 45$ & 68.4 & 10:00 & 66.5 & $7: 45$ & 99.9 \\
\hline & Average & 75.55 & & 75.95 & & 67.2 & & 67.3 & & 66.5 & & 98.1 \\
\hline
\end{tabular}

Table 13: Noise level data at Dilsukhnagar 
Published Online July 2019 in IJEAST (http://www.ijeast.com)

Table 14: Noise level data at Shaikhpet

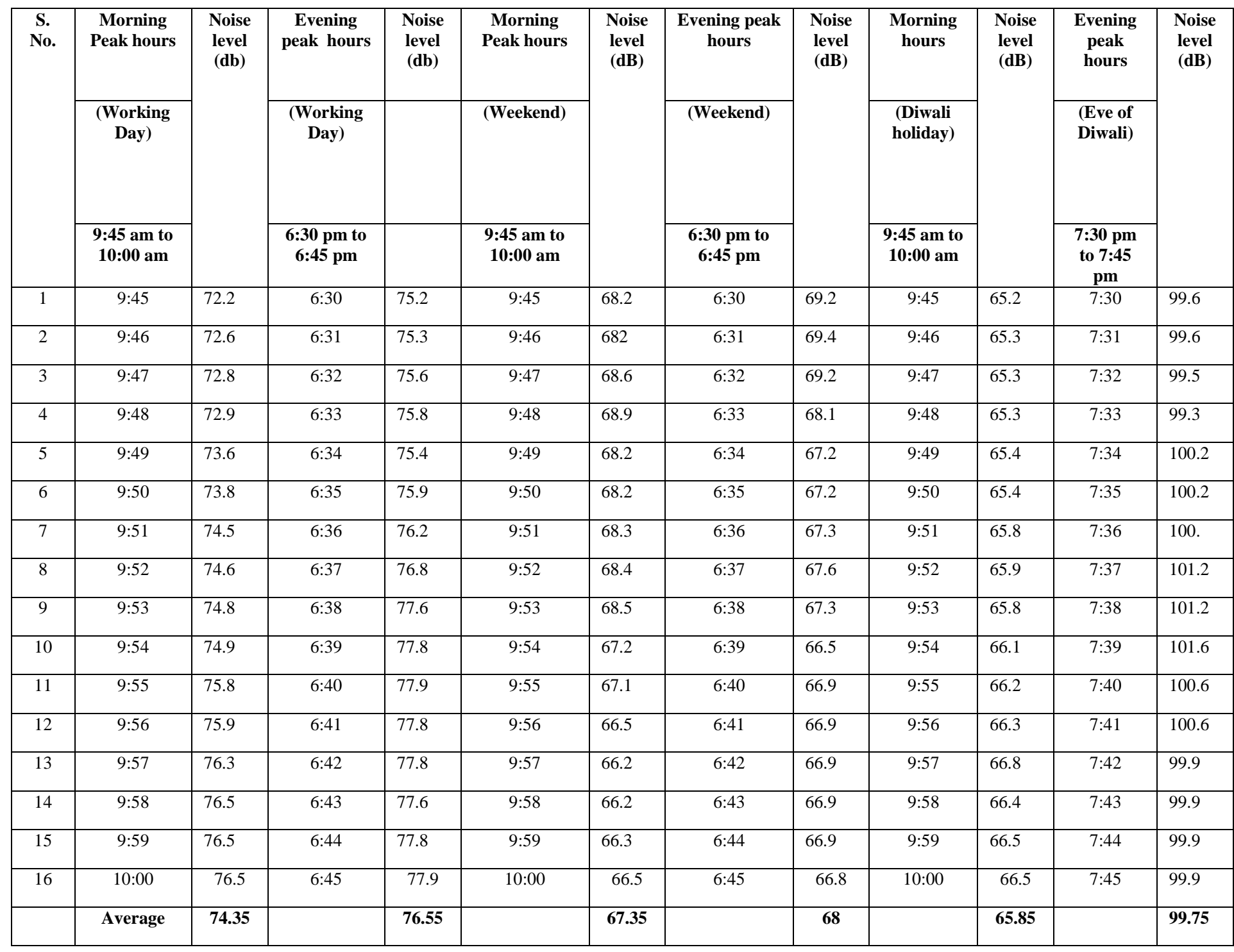


Published Online July 2019 in IJEAST (http://www.ijeast.com)

Table 15: Noise level data at Gachibowli

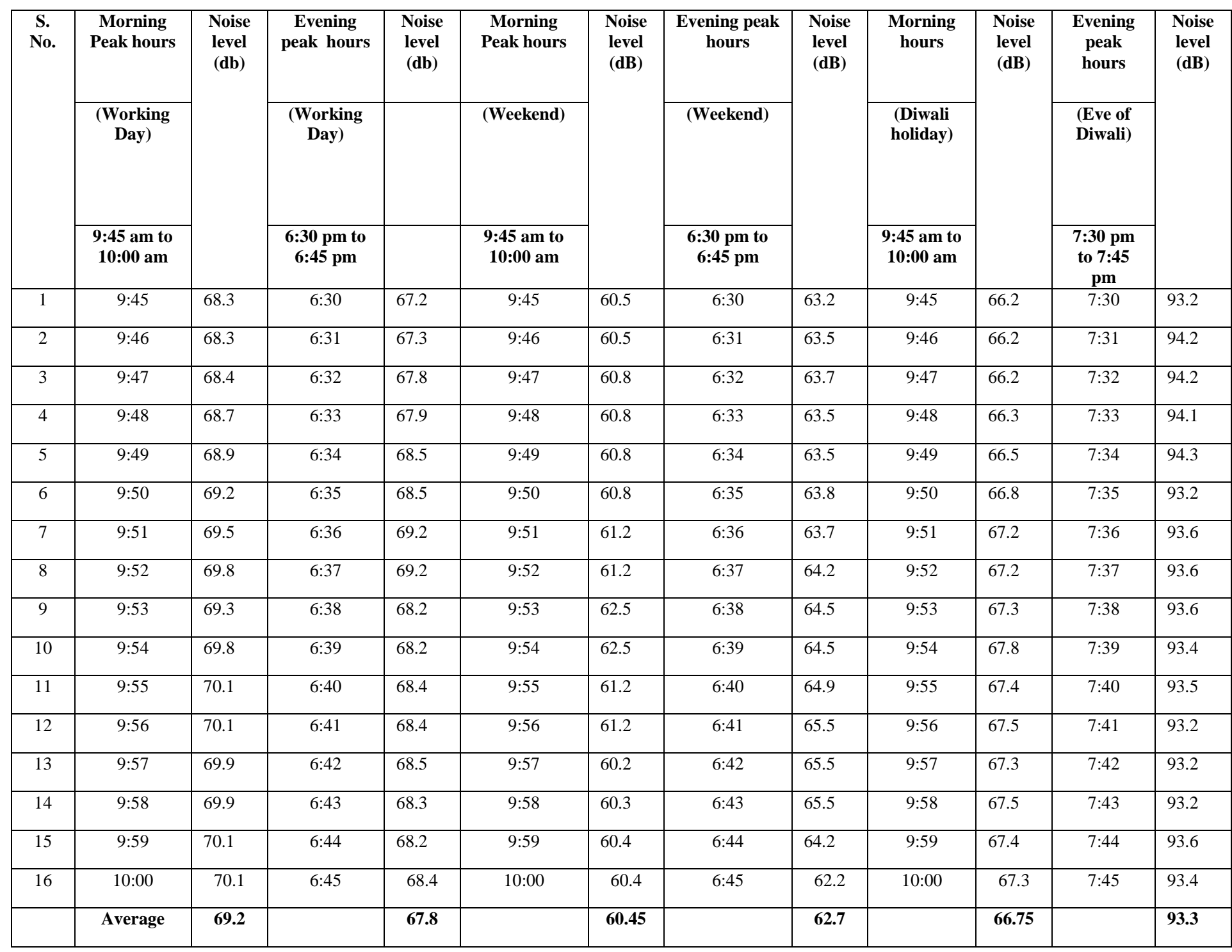


International Journal of Engineering Applied Sciences and Technology, 2019

Vol. 4, Issue 3, ISSN No. 2455-2143, Pages 573-605

Published Online July 2019 in IJEAST (http://www.ijeast.com)

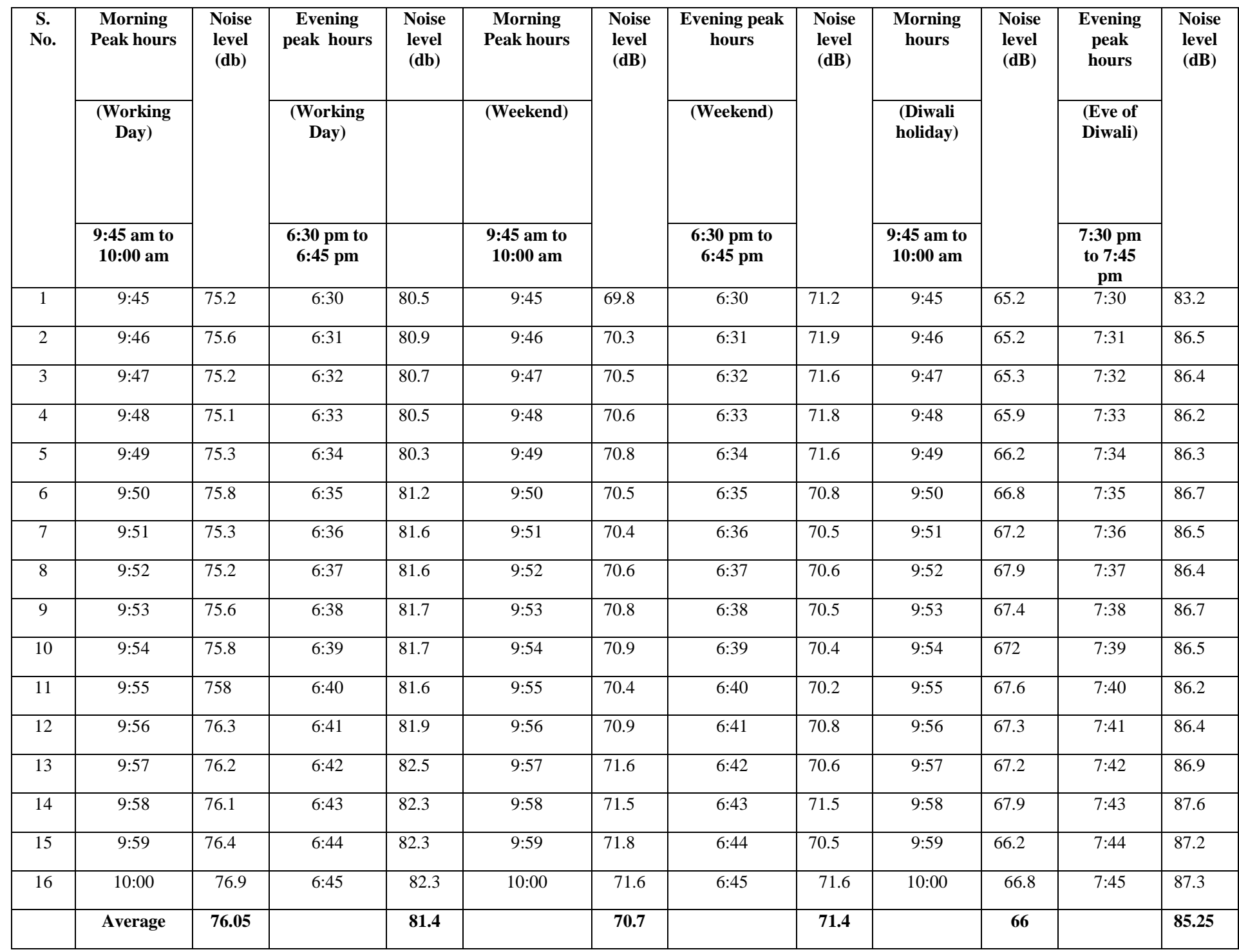

Table 16: Noise level data at Banjara Hills 
International Journal of Engineering Applied Sciences and Technology, 2019

Vol. 4, Issue 3, ISSN No. 2455-2143, Pages 573-605

Published Online July 2019 in IJEAST (http://www.ijeast.com)

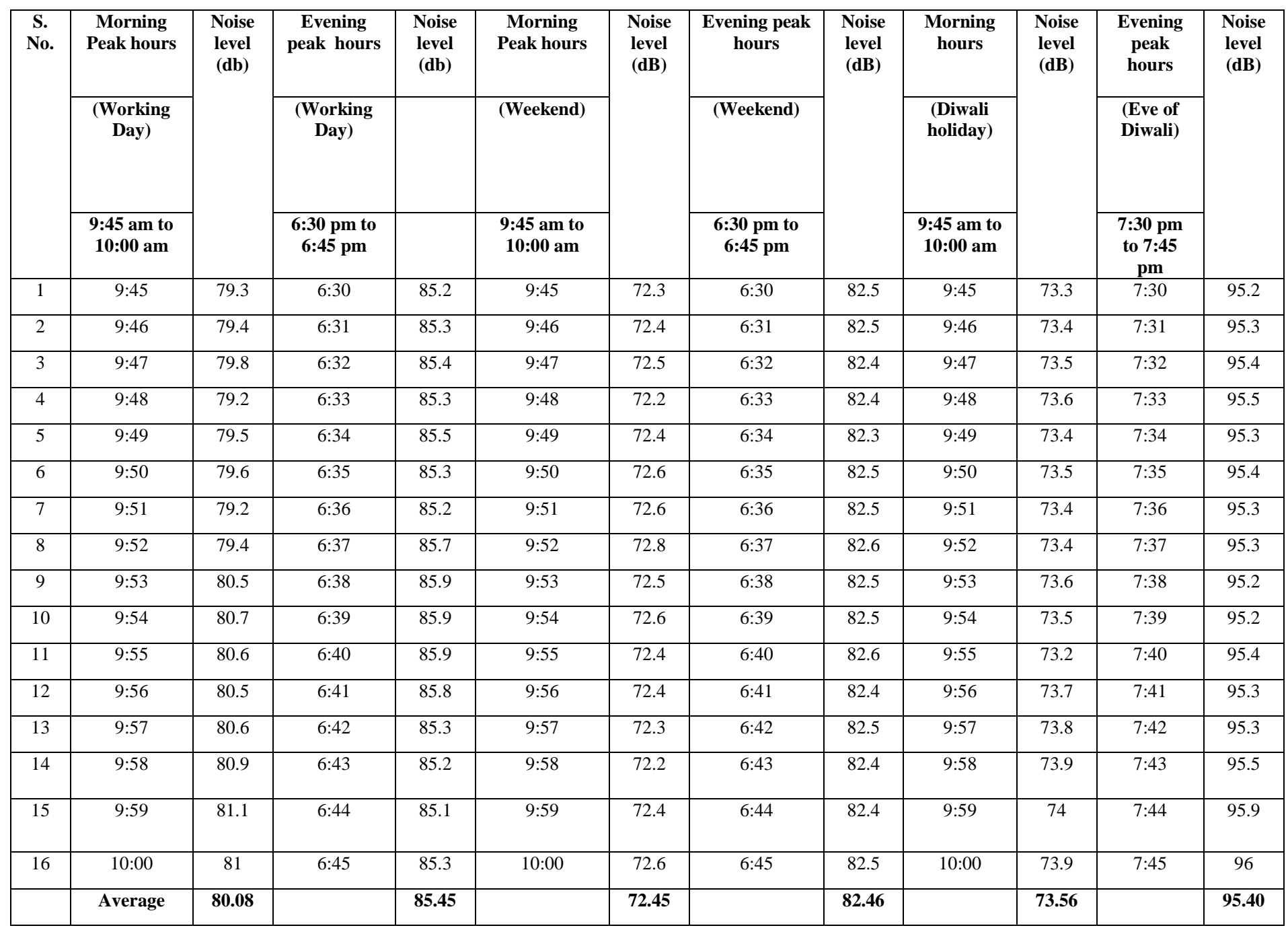

Table 17: Noise level data at Ameerpet 
Published Online July 2019 in IJEAST (http://www.ijeast.com)

Table 18: Noise level data at Bowenpally

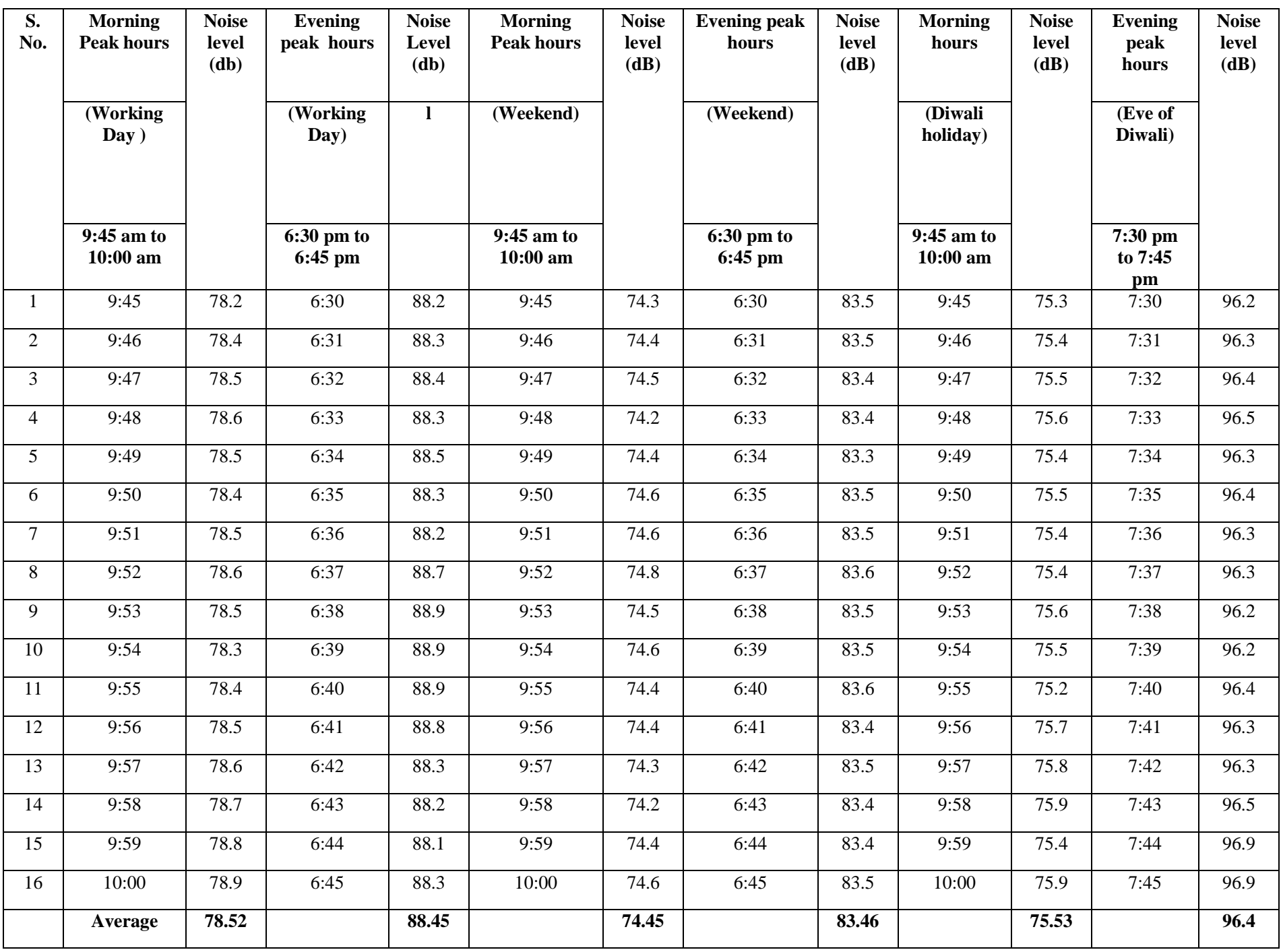


International Journal of Engineering Applied Sciences and Technology, 2019

Vol. 4, Issue 3, ISSN No. 2455-2143, Pages 573-605

Published Online July 2019 in IJEAST (http://www.ijeast.com)

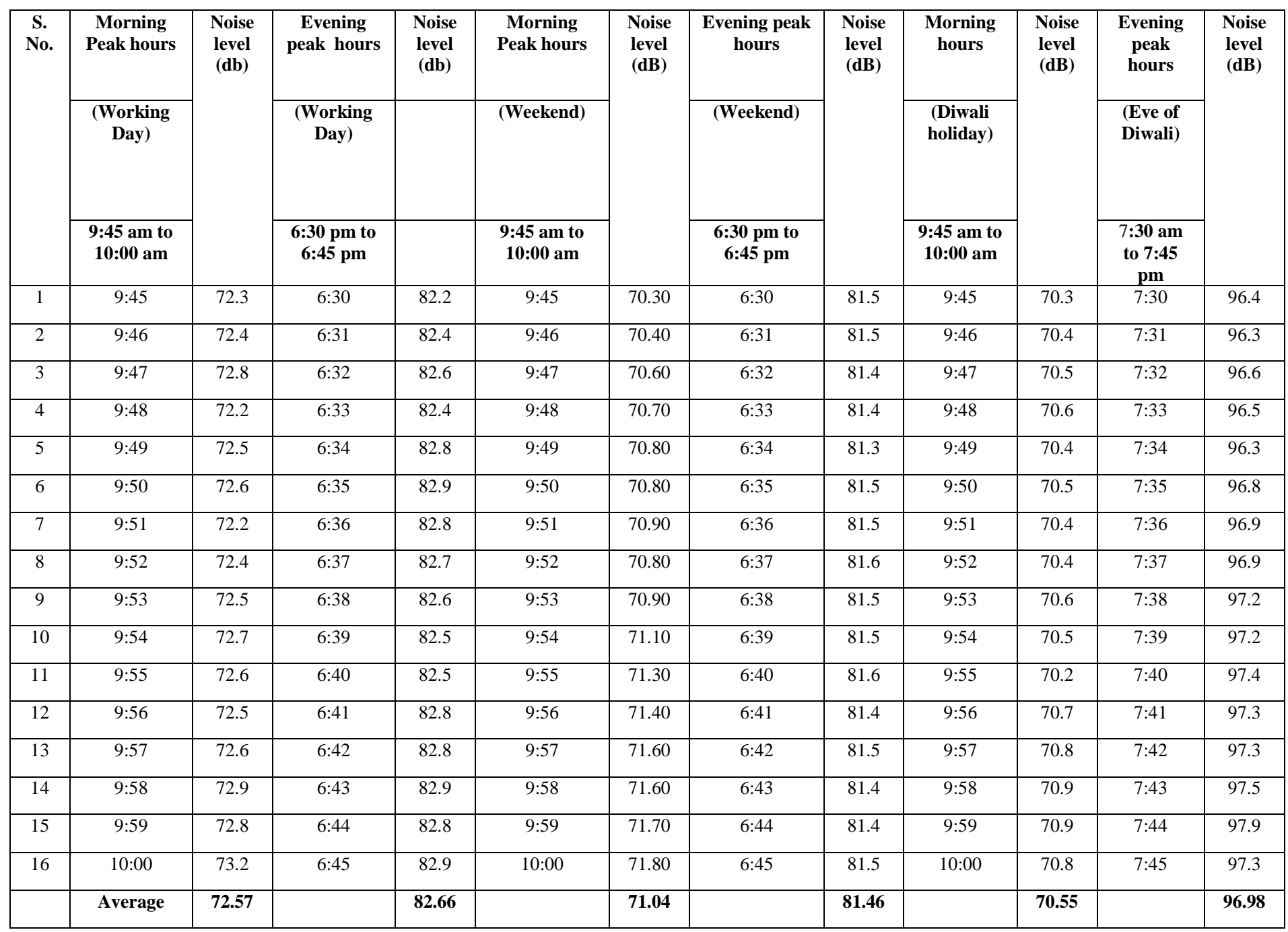

Table 19: Noise level data at Tirumalagiri 
International Journal of Engineering Applied Sciences and Technology, 2019

Vol. 4, Issue 3, ISSN No. 2455-2143, Pages 573-605

Published Online July 2019 in IJEAST (http://www.ijeast.com)

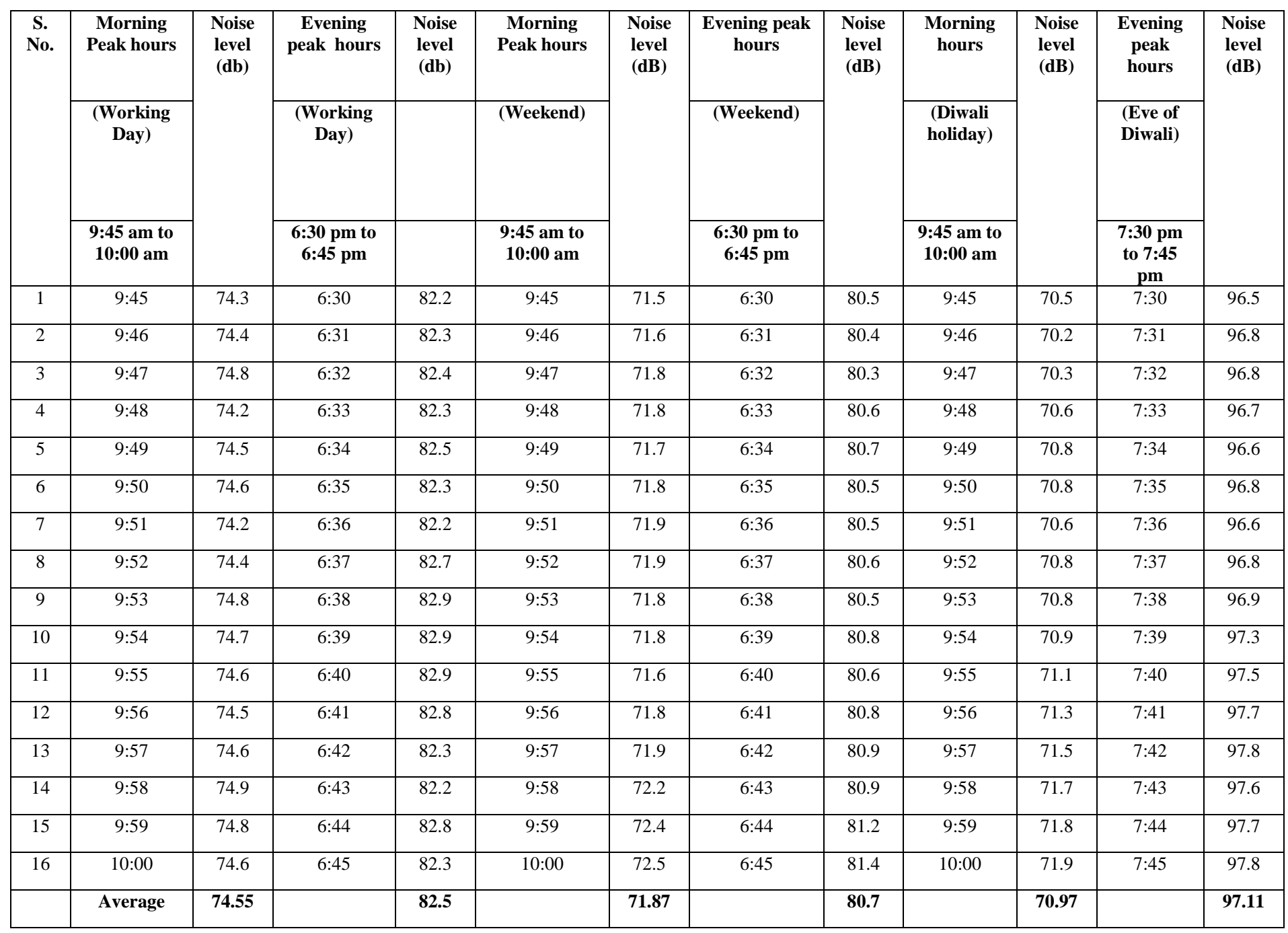

Table 20: Noise level data at Marredpally 
International Journal of Engineering Applied Sciences and Technology, 2019

Vol. 4, Issue 3, ISSN No. 2455-2143, Pages 573-605

Published Online July 2019 in IJEAST (http://www.ijeast.com)

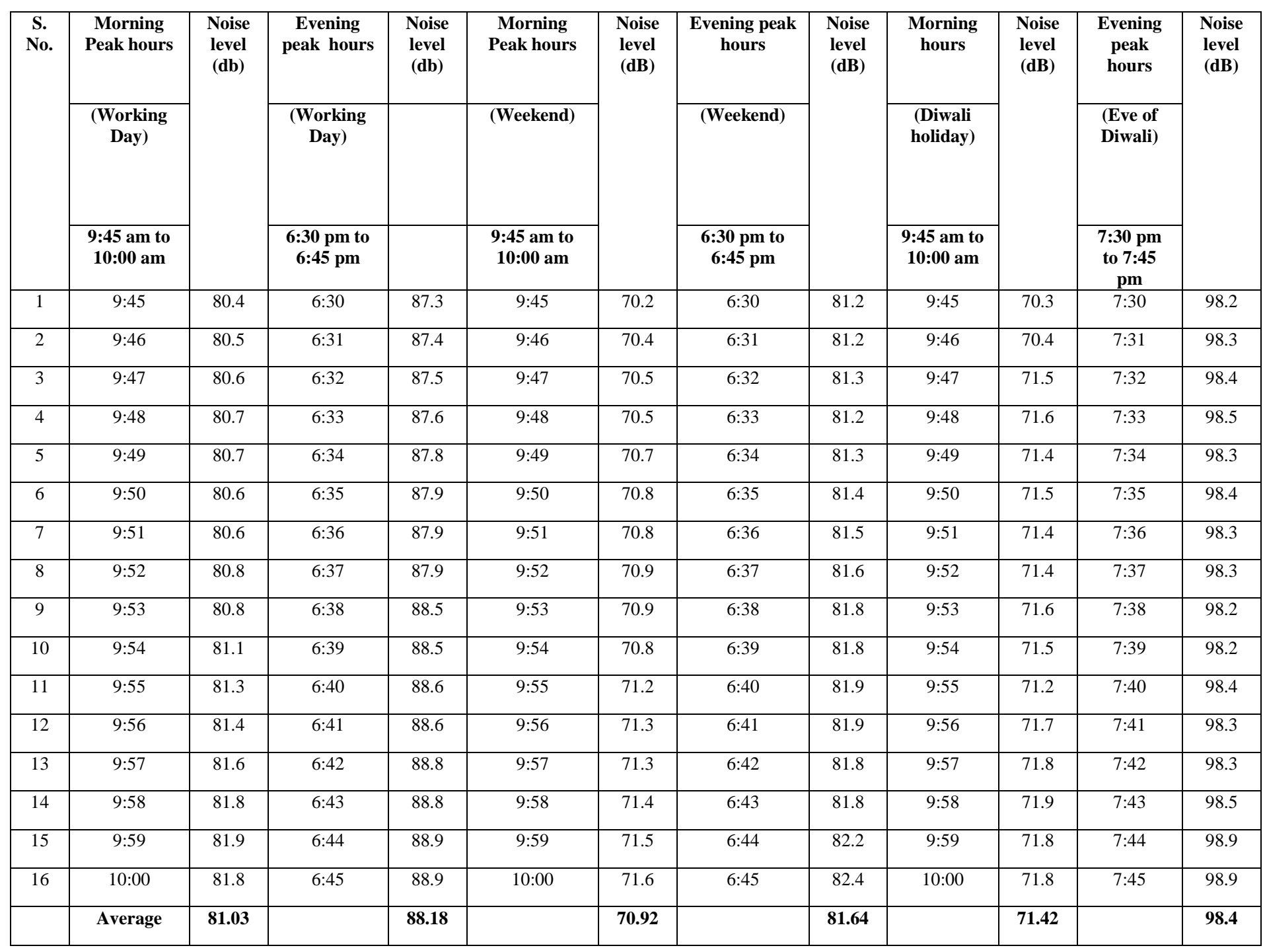

Table 21: Noise level data at Lalaguda 
Published Online July 2019 in IJEAST (http://www.ijeast.com)

Table 22: Noise level data at Amberpet

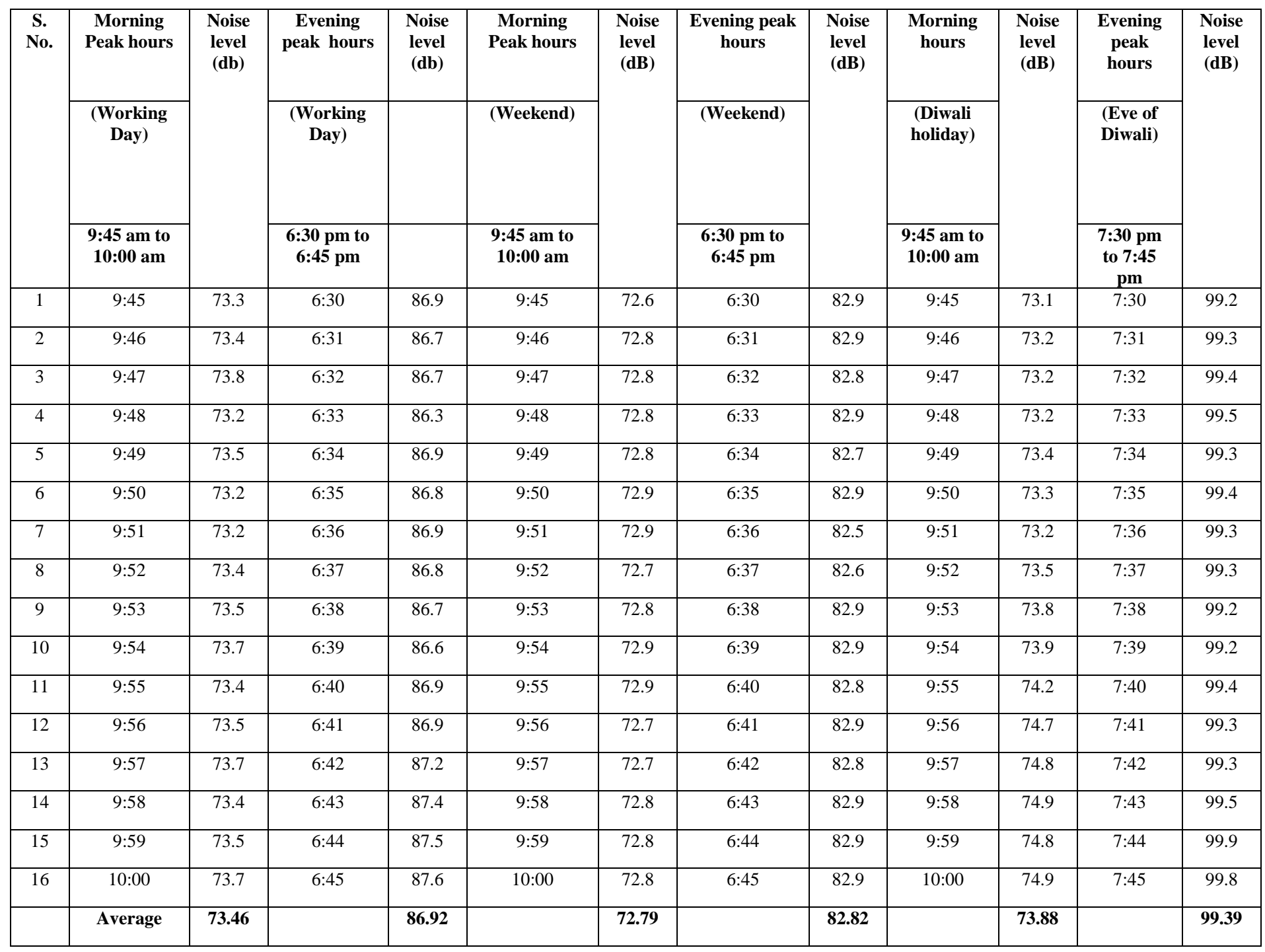


Published Online July 2019 in IJEAST (http://www.ijeast.com)

Table 23: Noise level data at Malakpet

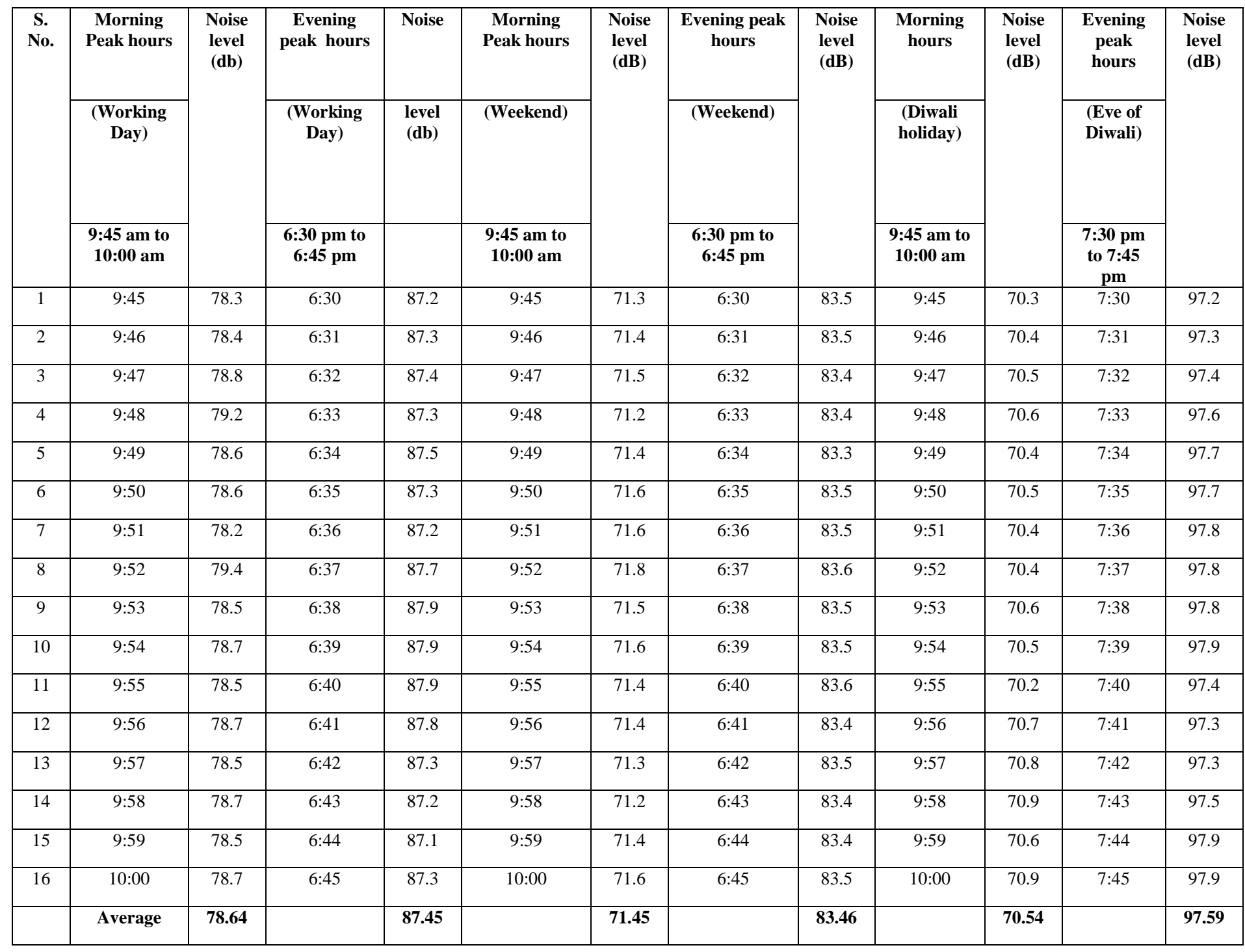


International Journal of Engineering Applied Sciences and Technology, 2019

Vol. 4, Issue 3, ISSN No. 2455-2143, Pages 573-605

Published Online July 2019 in IJEAST (http://www.ijeast.com)

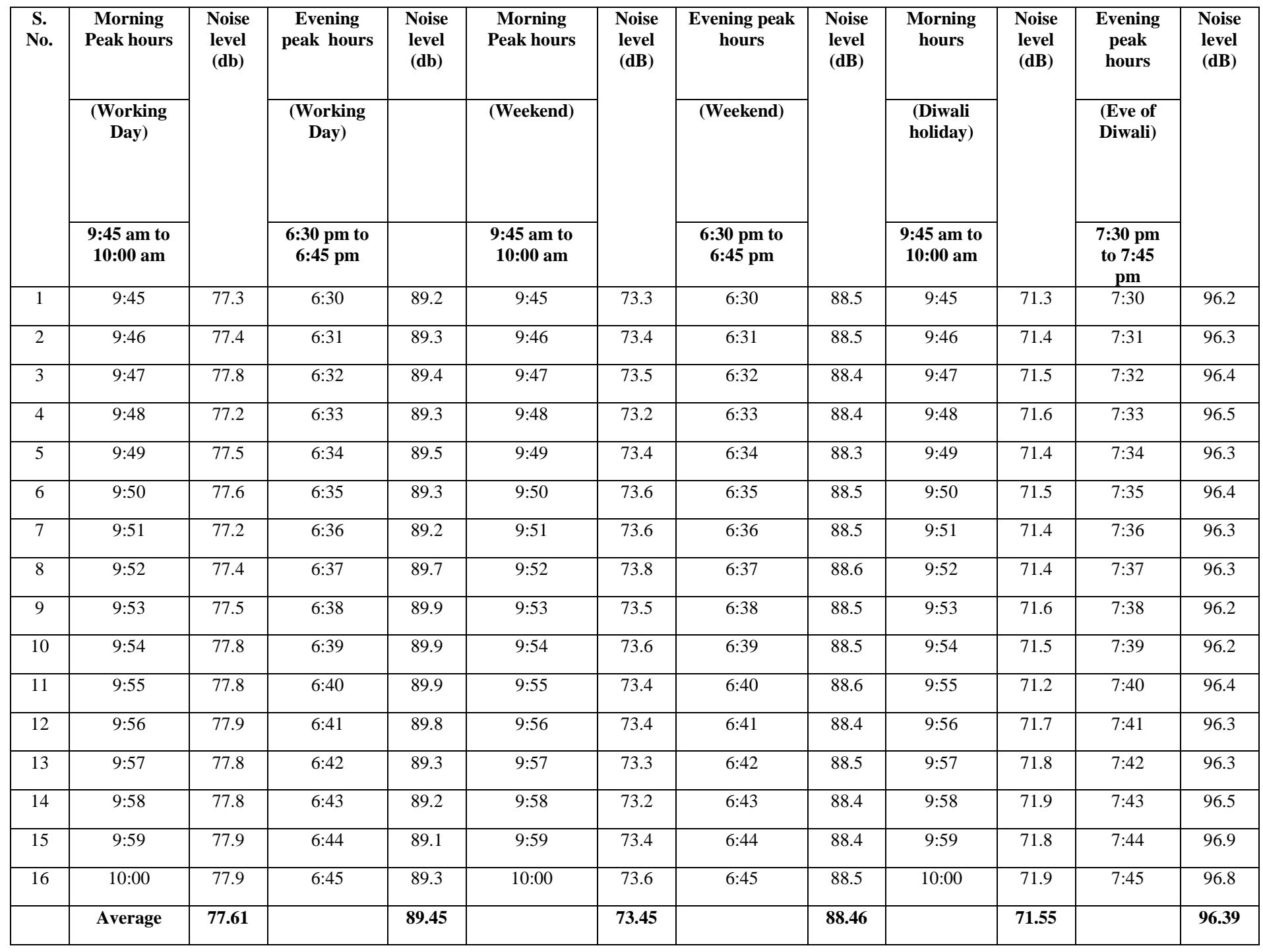

Table 24: Noise level data at Himayat Nagar 
International Journal of Engineering Applied Sciences and Technology, 2019

Vol. 4, Issue 3, ISSN No. 2455-2143, Pages 573-605

Published Online July 2019 in IJEAST (http://www.ijeast.com)

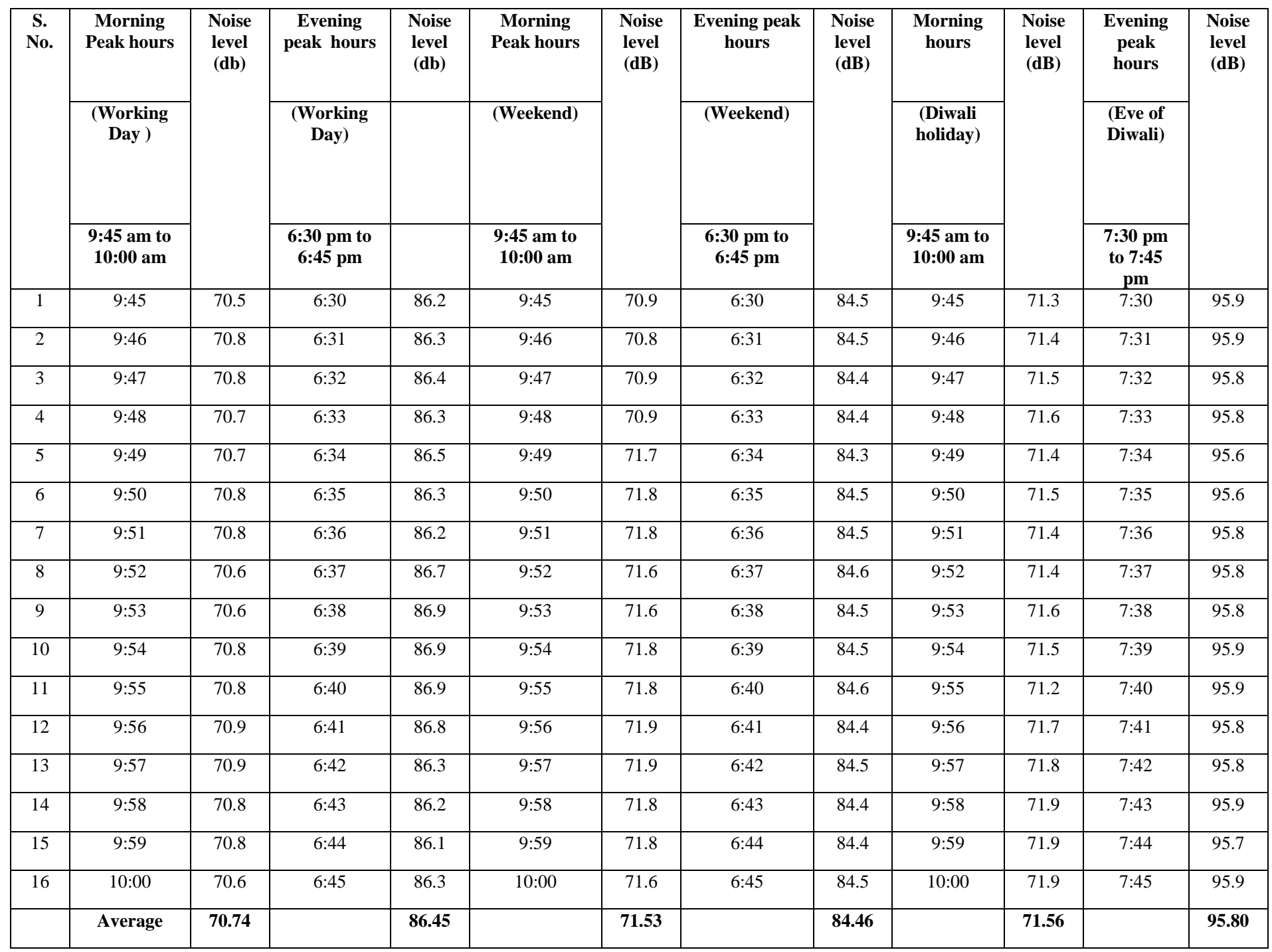

Table 25: Noise level data at Langer House 
International Journal of Engineering Applied Sciences and Technology, 2019

Vol. 4, Issue 3, ISSN No. 2455-2143, Pages 573-605

Published Online July 2019 in IJEAST (http://www.ijeast.com)

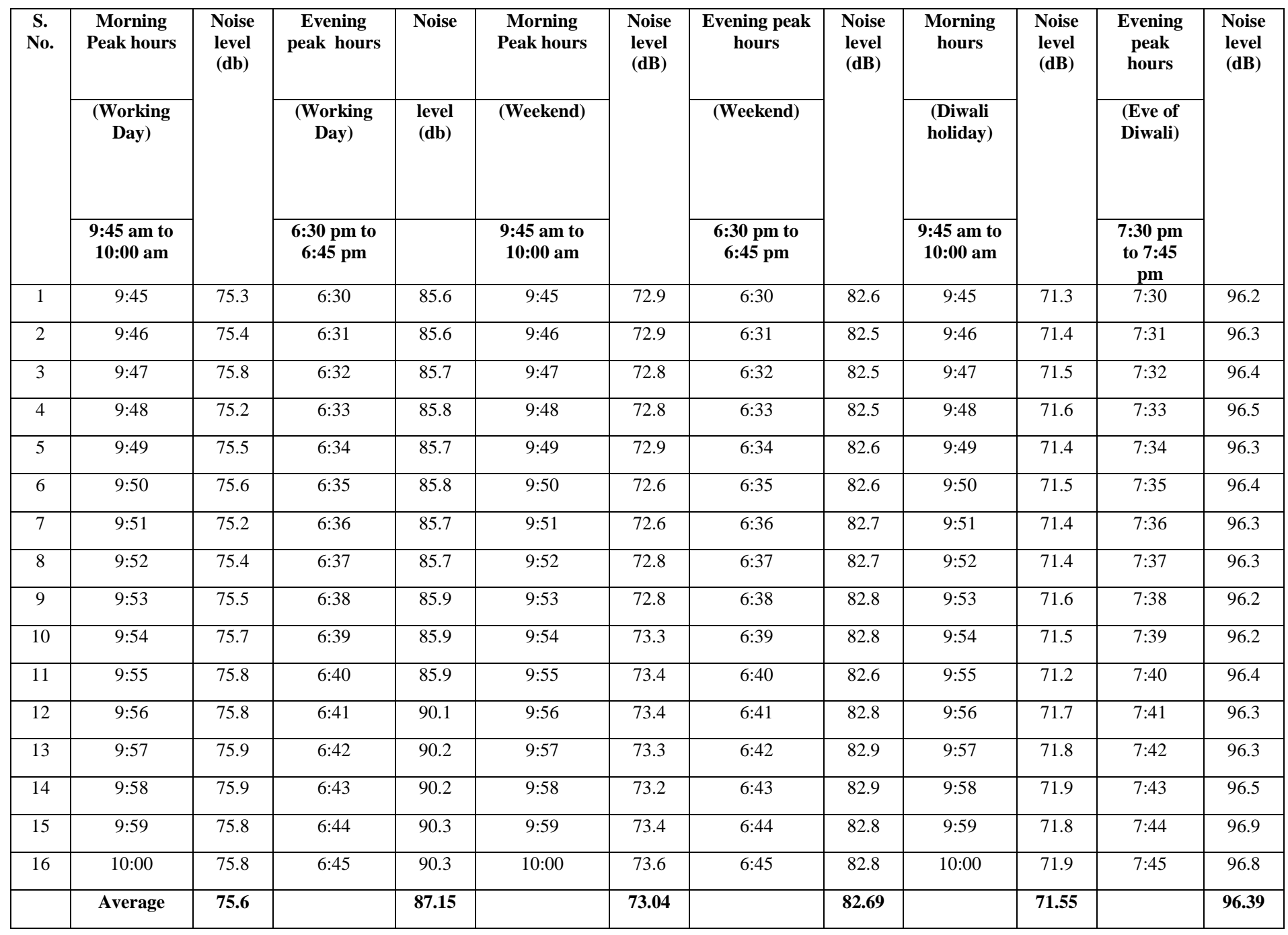

Table 26: Noise level data at Musheerabad 
International Journal of Engineering Applied Sciences and Technology, 2019

Vol. 4, Issue 3, ISSN No. 2455-2143, Pages 573-605

Published Online July 2019 in IJEAST (http://www.ijeast.com)

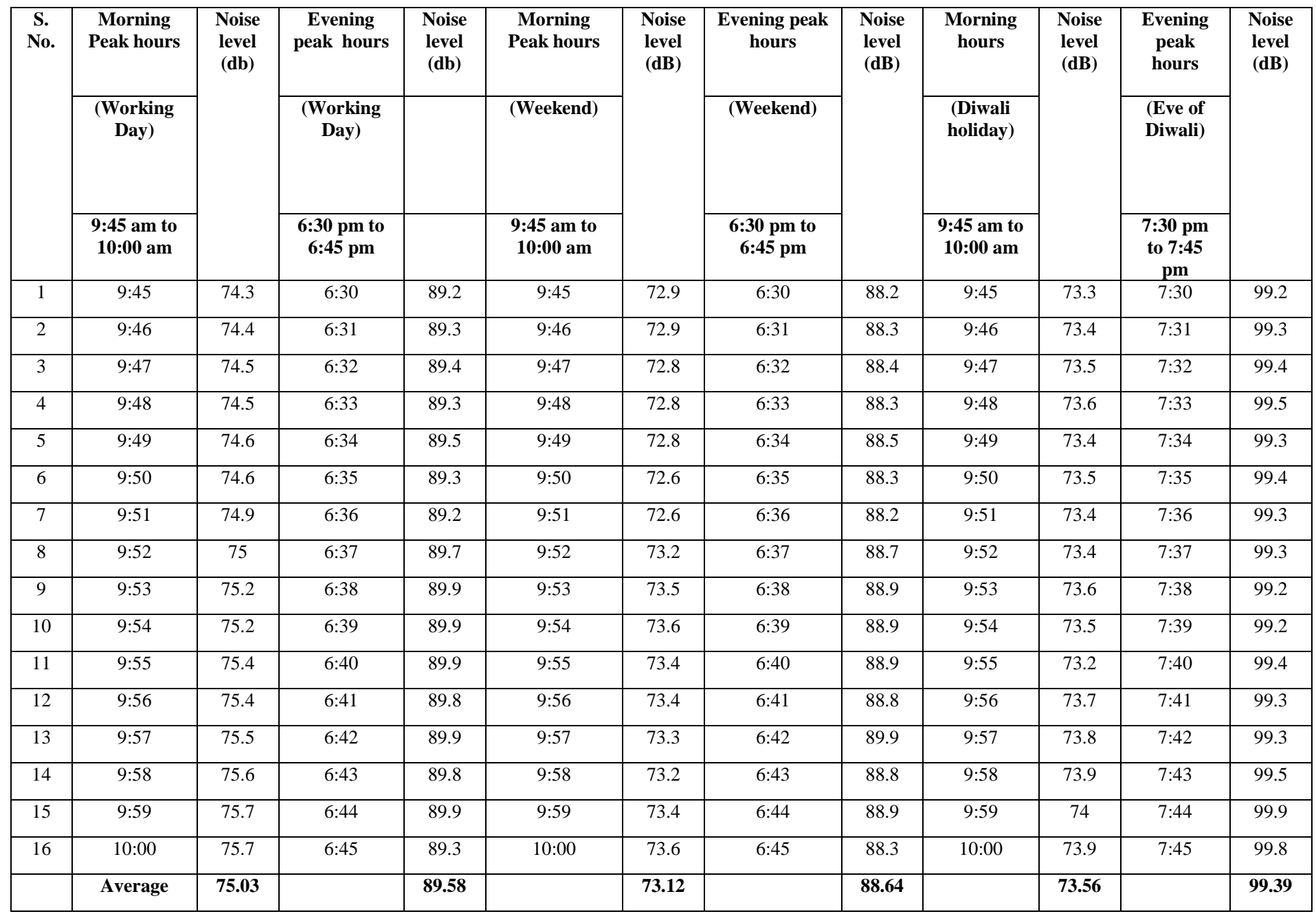

Table 27: Noise level data at Secunderabad 
Published Online July 2019 in IJEAST (http://www.ijeast.com)

\begin{tabular}{|c|c|c|c|c|c|c|c|c|c|c|c|c|}
\hline \multirow[t]{3}{*}{$\begin{array}{c}\text { S. } \\
\text { No. }\end{array}$} & $\begin{array}{c}\text { Morning } \\
\text { Peak hours }\end{array}$ & \multirow[t]{3}{*}{$\begin{array}{c}\text { Noise } \\
\text { level } \\
\text { (db) }\end{array}$} & $\begin{array}{c}\text { Evening } \\
\text { peak hours }\end{array}$ & $\begin{array}{c}\text { Noise } \\
\text { level } \\
\text { (db) }\end{array}$ & $\begin{array}{c}\text { Morning } \\
\text { Peak hours }\end{array}$ & \multirow[t]{3}{*}{$\begin{array}{l}\text { Noise } \\
\text { level } \\
\text { (dB) }\end{array}$} & $\begin{array}{c}\text { Evening peak } \\
\text { hours }\end{array}$ & \multirow[t]{3}{*}{$\begin{array}{l}\text { Noise } \\
\text { level } \\
\text { (dB) }\end{array}$} & $\begin{array}{c}\text { Morning } \\
\text { hours }\end{array}$ & \multirow[t]{3}{*}{$\begin{array}{c}\text { Noise } \\
\text { level } \\
\text { (dB) }\end{array}$} & $\begin{array}{c}\text { Evening } \\
\text { peak } \\
\text { hours }\end{array}$ & \multirow[t]{3}{*}{$\begin{array}{c}\text { Noise } \\
\text { level } \\
\text { (dB) }\end{array}$} \\
\hline & $\begin{array}{l}\text { (Working } \\
\text { Day) }\end{array}$ & & $\begin{array}{c}\text { (Working } \\
\text { Day) }\end{array}$ & & (Weekend) & & (Weekend) & & $\begin{array}{c}\text { (Diwali } \\
\text { holiday) }\end{array}$ & & $\begin{array}{l}\text { (Eve of } \\
\text { Diwali) }\end{array}$ & \\
\hline & $\begin{array}{c}9: 45 \text { am to } \\
10: 00 \text { am }\end{array}$ & & $\begin{array}{c}6: 30 \mathrm{pm} \text { to } \\
6: 45 \mathrm{pm}\end{array}$ & & $\begin{array}{c}\text { 9:45 am to } \\
10: 00 \text { am }\end{array}$ & & $\begin{array}{c}6: 30 \mathrm{pm} \text { to } \\
6: 45 \mathrm{pm}\end{array}$ & & $\begin{array}{c}\text { 9:45 am to } \\
10: 00 \text { am }\end{array}$ & & $\begin{array}{c}7: 30 \mathrm{pm} \\
\text { to } 7: 45 \\
\text { pm }\end{array}$ & \\
\hline 1 & $9: 45$ & 76.3 & $6: 30$ & 83.2 & $9: 45$ & 72.3 & $6: 30$ & 80.5 & $9: 45$ & 73.3 & $7: 30$ & 96.2 \\
\hline 2 & $9: 46$ & 76.4 & $6: 31$ & 83.4 & $9: 46$ & 72.4 & $6: 31$ & 80.5 & $9: 46$ & 73.4 & $7: 31$ & 96.3 \\
\hline 3 & $9: 47$ & 76.8 & $6: 32$ & 83.4 & $9: 47$ & 72.5 & $6: 32$ & 80.4 & $9: 47$ & 73.5 & $7: 32$ & 96.4 \\
\hline 4 & $9: 48$ & 76.2 & $6: 33$ & 83.3 & $9: 48$ & 72.2 & $6: 33$ & 80.4 & $9: 48$ & 73.6 & $7: 33$ & 96.5 \\
\hline 5 & $9: 49$ & 76.5 & $6: 34$ & 83.5 & $9: 49$ & 72.4 & $6: 34$ & 80.3 & $9: 49$ & 73.4 & $7: 34$ & 96.3 \\
\hline 6 & $9: 50$ & 76.6 & $6: 35$ & 83.3 & $9: 50$ & 72.6 & $6: 35$ & 80.5 & $9: 50$ & 73.5 & $7: 35$ & 96.4 \\
\hline 7 & $9: 51$ & 76.2 & $6: 36$ & 83.2 & $9: 51$ & 72.6 & $6: 36$ & 80.5 & $9: 51$ & 73.4 & $7: 36$ & 96.3 \\
\hline 8 & $9: 52$ & 76.4 & $6: 37$ & 83.7 & $9: 52$ & 72.8 & $6: 37$ & 80.6 & $9: 52$ & 73.4 & $7: 37$ & 96.3 \\
\hline 9 & $9: 53$ & 76.5 & $6: 38$ & 83.9 & $9: 53$ & 72.5 & $6: 38$ & 80.5 & $9: 53$ & 73.6 & $7: 38$ & 96.2 \\
\hline 10 & $9: 54$ & 76.7 & $6: 39$ & 83.9 & $9: 54$ & 72.6 & $6: 39$ & 80.5 & $9: 54$ & 73.5 & $7: 39$ & 96.2 \\
\hline 11 & $9: 55$ & 76.6 & $6: 40$ & 83.9 & $9: 55$ & 72.4 & $6: 40$ & 80.6 & $9: 55$ & 73.2 & $7: 40$ & 96.4 \\
\hline 12 & $9: 56$ & 76.5 & $6: 41$ & 83.8 & $9: 56$ & 72.4 & $6: 41$ & 80.4 & $9: 56$ & 73.7 & $7: 41$ & 96.3 \\
\hline 13 & $9: 57$ & 76.6 & $6: 42$ & 83.3 & $9: 57$ & 72.3 & $6: 42$ & 80.5 & $9: 57$ & 73.8 & $7: 42$ & 96.3 \\
\hline 14 & $9: 58$ & 76.9 & $6: 43$ & 83.2 & $9: 58$ & 72.2 & $6: 43$ & 80.4 & $9: 58$ & 73.9 & $7: 43$ & 96.5 \\
\hline 15 & $9: 59$ & 76.8 & $6: 44$ & 83.1 & $9: 59$ & 72.4 & $6: 44$ & 80.4 & $9: 59$ & 74 & $7: 44$ & 95.9 \\
\hline \multirow[t]{2}{*}{16} & $10: 00$ & 76.9 & $6: 45$ & 83.3 & $10: 00$ & 72.6 & $6: 45$ & 80.5 & $10: 00$ & 73.9 & $7: 45$ & 96.2 \\
\hline & Average & 76.55 & & 83.46 & & 72.45 & & 80.46 & & 73.56 & & 96.29 \\
\hline
\end{tabular}

Table 28: Noise level data at Bahadurpura 
Published Online July 2019 in IJEAST (http://www.ijeast.com)

\begin{tabular}{|c|c|c|c|c|c|c|c|c|c|c|c|c|}
\hline \multirow[t]{2}{*}{$\begin{array}{l}\text { S. } \\
\text { No. }\end{array}$} & $\begin{array}{c}\text { Morning } \\
\text { Peak hours }\end{array}$ & $\begin{array}{l}\text { Noise } \\
\text { level } \\
\text { (db) }\end{array}$ & $\begin{array}{c}\text { Evening } \\
\text { peak hours }\end{array}$ & Noise & $\begin{array}{c}\text { Morning } \\
\text { Peak hours }\end{array}$ & \multirow[t]{2}{*}{$\begin{array}{l}\text { Noise } \\
\text { level } \\
\text { (dB) }\end{array}$} & $\begin{array}{l}\text { Evening peak } \\
\text { hours }\end{array}$ & \multirow[t]{2}{*}{$\begin{array}{l}\text { Noise } \\
\text { level } \\
\text { (dB) }\end{array}$} & $\begin{array}{c}\text { Morning } \\
\text { hours }\end{array}$ & \multirow[t]{2}{*}{$\begin{array}{l}\text { Noise } \\
\text { level } \\
\text { (dB) }\end{array}$} & $\begin{array}{c}\text { Evening } \\
\text { peak } \\
\text { hours }\end{array}$ & \multirow[t]{2}{*}{$\begin{array}{l}\text { Noise } \\
\text { level } \\
\text { (dB) }\end{array}$} \\
\hline & $\begin{array}{c}\text { (Working } \\
\text { Day) }\end{array}$ & & $\begin{array}{c}\text { (Working } \\
\text { Day) }\end{array}$ & $\begin{array}{l}\text { level } \\
(\text { db) }\end{array}$ & (Weekend) & & (Weekend) & & $\begin{array}{l}\text { (Diwali } \\
\text { holiday) }\end{array}$ & & $\begin{array}{l}\text { (Eve of } \\
\text { Diwali) }\end{array}$ & \\
\hline 1 & $9: 45$ & 70.3 & $6: 30$ & 81.2 & $9: 45$ & 69.3 & $6: 30$ & 82.5 & $9: 45$ & 73.3 & $7: 30$ & 94.2 \\
\hline 2 & 9:46 & 70.4 & $6: 31$ & 81.3 & $9: 46$ & 69.4 & $6: 31$ & 82.5 & $9: 46$ & 73.4 & $7: 31$ & 94.3 \\
\hline 3 & $9: 47$ & 70.8 & $6: 32$ & 81.4 & $9: 47$ & 69.4 & $6: 32$ & 82.4 & $9: 47$ & 73.5 & $7: 32$ & 94.4 \\
\hline 7 & $9: 51$ & 70.2 & $6: 36$ & 81.2 & $9: 51$ & 69.6 & $6: 36$ & 82.5 & $9: 51$ & 73.4 & $7: 36$ & 94.3 \\
\hline 8 & $9: 52$ & 70.4 & $6: 37$ & 81.7 & $9: 52$ & 69.6 & $6: 37$ & 82.6 & $9: 52$ & 73.4 & $7: 37$ & 94.3 \\
\hline 9 & $\begin{array}{l}9: 53 \\
\end{array}$ & $\begin{array}{l}70.5 \\
\end{array}$ & 6:38 & 81.9 & $\begin{array}{l}9: 53 \\
\end{array}$ & 69.7 & $\begin{array}{ll}6: 38 \\
\end{array}$ & 82.5 & $\begin{array}{l}9: 53 \\
\end{array}$ & 73.6 & 7:38 & 94.2 \\
\hline 10 & $9: 54$ & 70.7 & $6: 39$ & 81.9 & $9: 54$ & 69.7 & $6: 39$ & 82.5 & $9: 54$ & 73.5 & $7: 39$ & 94.2 \\
\hline 11 & $9: 55$ & 70.6 & $6: 40$ & 81.9 & $9: 55$ & 69.8 & $6: 40$ & 82.6 & $9: 55$ & 73.2 & $7: 40$ & 94.4 \\
\hline 12 & $9: 56$ & 70.5 & $6: 41$ & 81.8 & $9: 56$ & 69.8 & $6: 41$ & 82.4 & $9: 56$ & 73.7 & $7: 41$ & 94.3 \\
\hline 13 & $9: 57$ & 70.6 & $6: 42$ & 81.3 & $9: 57$ & 69.8 & $6: 42$ & 82.5 & $9: 57$ & 73.8 & $7: 42$ & 94.3 \\
\hline 14 & $9: 58$ & 70.9 & $6: 43$ & 81.2 & $9: 58$ & 69.8 & $6: 43$ & 82.4 & $9: 58$ & 73.9 & $7: 43$ & 94.5 \\
\hline
\end{tabular}

Table 29: Noise level data at Bandlaguda 
International Journal of Engineering Applied Sciences and Technology, 2019

Vol. 4, Issue 3, ISSN No. 2455-2143, Pages 573-605

Published Online July 2019 in IJEAST (http://www.ijeast.com)

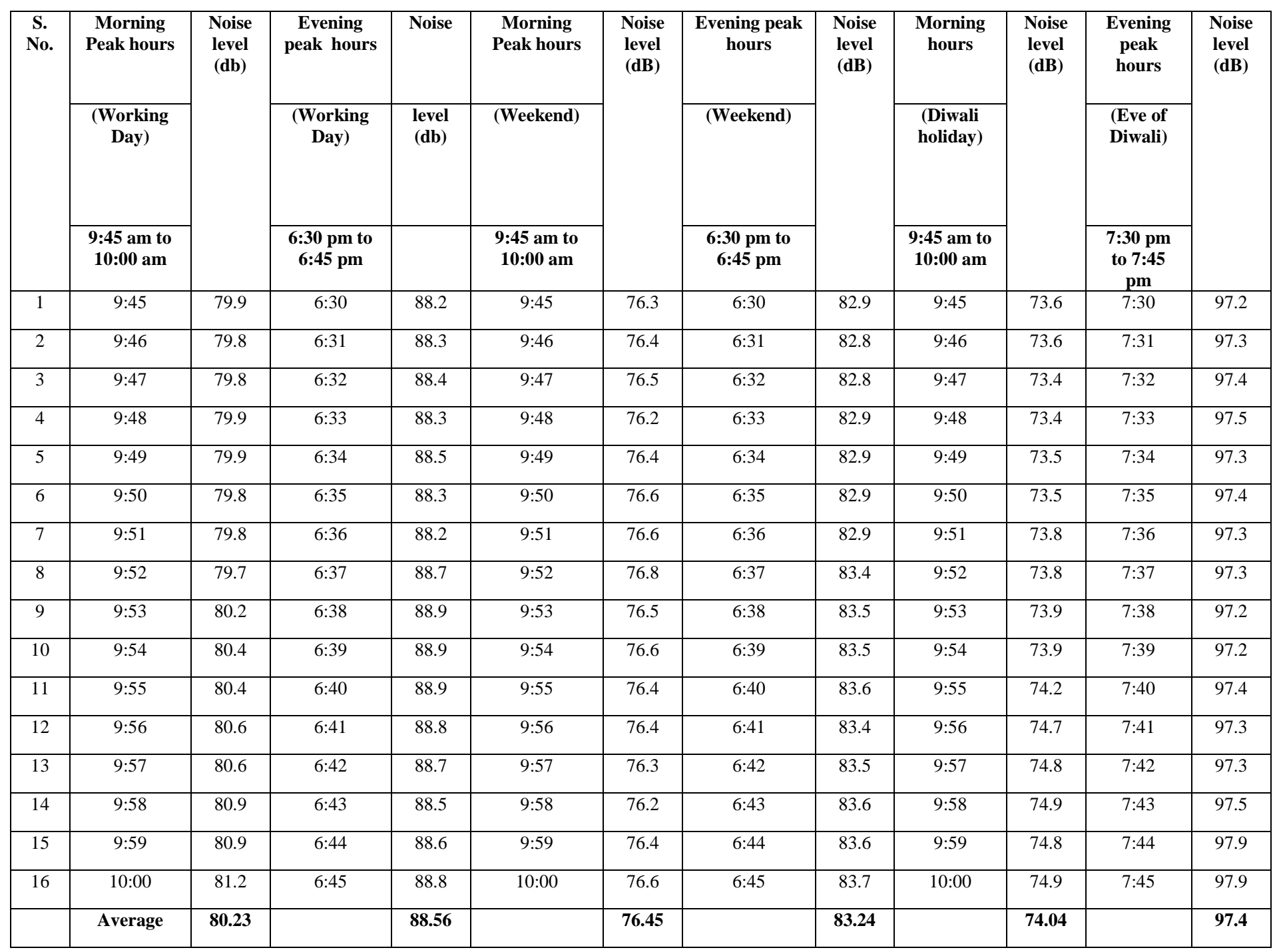

Table 30: Noise level data at Moosarambagh 


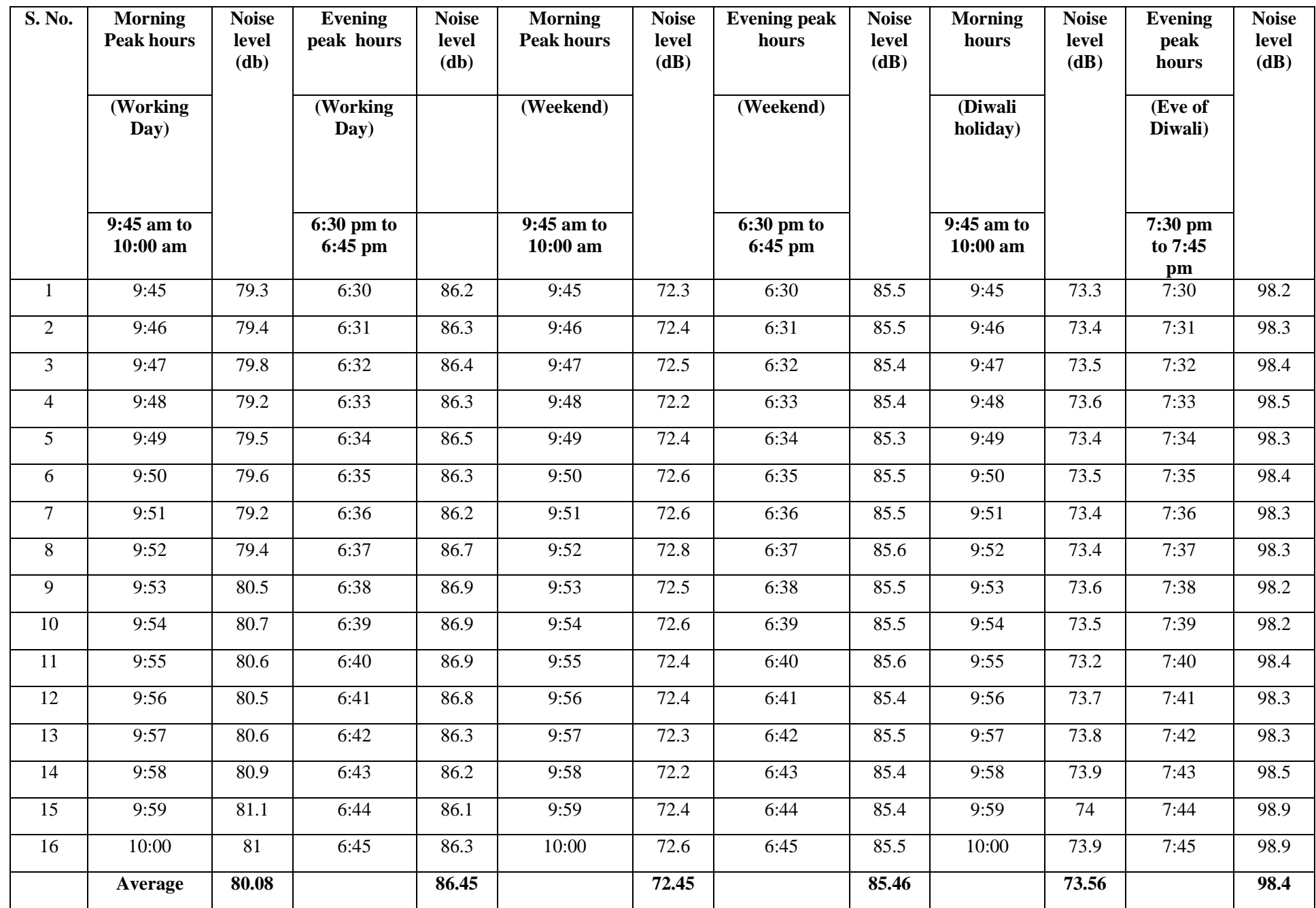

\section{IV.CONCLUSION}

This study is basically a survey data of noise level in major areas of Hyderabad like Charminar, Lad Bazaar, Afzal gunj, Mir alam mandi, Begum Bazaar, Sultan Bazaar, Kothi, Abids, Mehdipatnam, Nampally, Lakdi-ka-pool, Dilsukhnagar,

\section{Table 31: Noise level data at Khairtabad}

Shaikpet, Gachibowli, Banjara Hills, Ameerpet, Bowenpally, Tirumalagiri, Marredpally, Lalaguda, Amberpet, Malakpet, Himayat Nagar, Langer house, Musheerabad, Secunderabad, Bahadurpura, Bandlaguda, Moosarambagh, Khairtabad. These are heavily populated and commercial regions. Noise was recorded and compared for three different days: weekday, weekend, Diwali holiday (morning and evening) for consecutive 15 minutes. Data collected reveals that Diwali day the noise was maximum as compared to the regular days. This 


\section{International Journal of Engineering Applied Sciences and Technology, 2019 \\ Vol. 4, Issue 3, ISSN No. 2455-2143, Pages 573-605 \\ Published Online July 2019 in IJEAST (http://www.ijeast.com)}

data aims to spread awareness regarding noise pollution that usually people over look during the excitement of festivities.

\section{REFERENCE}

[1] Keerthana, Gobinath R., Neelima S., Chitravel V., Saranya S., and Kannan T. (2013). AnAnalysis of noise pollution in Tirupur city. Sch J Eng Tech., 1, (pp.154-168).

[2] Li B., Taoa S., Dawsona R.W., Caoa J., and Lamb K.A. (2002). GIS based road traffic noise prediction model. App Acoustics., 63, (pp.679-691).

[3] Wazir A. (2011). GIS based Assessment of Noise Pollution in Guwahati City of Assam, India. International Journal of Environmental Sciences. doi:10.6088/ijes.00202020034.

[4] Hammersen F., Niemann H., and Hoebel J. (2016). Environmental Noise Annoyance and Mental Health in Adults: Findings from the Cross-Sectional German Health Update (GEDA) Study 2021. Int. J. Environ. Res. Public Health. doi: 10.3390/ijerph13100954.

[5] Bhabananda P. and Kalyan K. (2013). An experimental study of noise pollution in Gauhati University campus, Guwahati, Assam, India. International Journal of Environmental Sciences. 3, (pp-5).

[6] Fyhri A. and Klæboe R. (2009). Road traffic`c noise, sensitivity, annoyance and self-reported health-A structural equation model exercisell. Environment International. 35, (pp.91-97).

[7] Ma J., Li C., Kwan M. and Chai Y. (2018). A Multilevel Analysis of Perceived Noise Pollution, Geographic Contexts and Mental Health in Beijing. Int. J. Environ. Res. Public

Health. 7, doi: 10.3390/ijerph15071479.

[8] Singh N. and Davar S. C. (2004). Noise PollutionSources, Effects and Control. J. Hum. Ecol. 16, (181-187).

[9] Romain S., Frédérique F. D. L. and Barbara L. (2019). Evidence of the environmental impact of noise pollution on biodiversity: a systematic map protocol. Environ Evid. 8, (pp8).

[10] Bernath-Plaisted J. and Koper N., (2016). Physical footprint of oil and gas infrastructure, not anthropogenic noise, reduces nesting success of some grassland songbirds. Biol Conserv. 204, (pp.434-41).

[11] Aditi S., Akanksha J. and Jhinuk C., (2013). Effect of sound on plant growth, Asian Journal of Plant Science and Research. 3, (pp.28-30).

[12] Collins M.E. and Foreman J.E.K. (2001). The Effect of Sound on the Growth of Plants. Canadian Acoustics. 29, (pp:3-8). 